\title{
Discordant and heterogeneous clinically relevant genomic alterations in circulating tumor cells versus plasma DNA from men with metastatic castration resistant prostate cancer
}

Santosh Gupta ${ }^{1,2}$, Daniel H. Hovelson ${ }^{3}$, Gabor Kemeny ${ }^{1}$, Susan Halabi ${ }^{4}$, Wen-Chi Foo $^{1}$, Monika Anand ${ }^{1}$, Jason A. Somarelli ${ }^{1,5}$, Scott A. Tomlins ${ }^{4}$, Emmanuel S. Antonarakis ${ }^{6}$, Jun Luo ${ }^{7}$, Ryan V. Dittamore ${ }^{8}$, Daniel J. George ${ }^{1}$, Colin Rothwell ${ }^{1}$, David M. Nanus ${ }^{5}$, Andrew J. Armstrong ${ }^{1,5^{*}}$, Simon G. Gregory ${ }^{1,2^{*}}$

\section{Affiliations:}

1. Duke Cancer Institute Center for Prostate and Urologic Cancers, Duke University, Durham NC USA.

2. Duke Molecular Physiology Institute, Duke University, Durham NC USA.

3. Michigan Center for Translational Pathology, University of Michigan, Ann Arbor MI USA.

4. Department of Biostatistics and Bioinformatics, Duke University, Durham, NC USA.

5. Departments of Medicine, Surgery, Pharmacology and Cancer Biology, Duke University, Durham NC USA.

6. Prostate Cancer Research Program, Sidney Kimmel Comprehensive Cancer Center, Johns Hopkins, Baltimore, MD USA.

7. James Buchanan Brady Urological Institute and the Department of Urology, Johns Hopkins University School of Medicine, Baltimore, MD USA.

8. Epic Sciences, La Jolla, California.

Corresponding author: Andrew J Armstrong and Simon G Gregory Contribution: * These authors contributed equally

Address: Duke University Medical Center Box 103861, Durham NC 27710, USA

E-mail: andrew.armstrong@duke.edu and simon.gregory@duke.edu

Fax: +1 (919) 613-3900 or +1 (919) 660-0178, Phone: +1 (919) 668-8797

Running head: Genomic discordance of CTC vs. cfDNA in MCRPC

This is the author manuscript accepted for publication and has undergone full peer review but has not been through the copyediting, typesetting, pagination and proofreading process, which may lead to differences between this version and the Version of Record. Please cite this article as doi: $10.1002 /$ gcc. 22824

This article is protected by copyright. All rights reserved. 
Conflict of interest disclosures: Andrew J. Armstrong reports research funding from Medivation/Astellas and Janssen as well as consulting for those two companies. The other authors disclosed no potential conflicts.

\begin{abstract}
Circulating tumor cell (CTC) and cell-free (cf) DNA-based genomic alterations are increasingly being used for clinical decision-making in oncology. However, the concordance and discordance between paired CTC and cfDNA genomic profiles remain largely unknown. We performed comparative genomic hybridization (CGH) on CTCs and cfDNA, and low-pass whole genome sequencing (IpWGS) on cfDNA to characterize genomic alterations (CNA) and tumor content in two independent prospective studies of 93 men with MCRPC treated with enzalutamide/abiraterone, or radium-223. Comprehensive analysis of 69 patient CTCs and 72 cfDNA samples from 93 men with $\mathrm{mCRPC}$, including 64 paired samples, identified common concordant gains in FOXA1, AR, and MYC, and losses in BRCA1, PTEN, and RB1 between CTCs and cfDNA. Concordant PTEN loss and discordant BRCA2 gain were associated with significantly worse outcomes in Epic AR-V7 negative men with mCRPC treated with abiraterone/enzalutamide. We identified and externally validated CTC-specific genomic alternations that were discordant in paired cfDNA, even in samples with high tumor content. These CTC/cfDNA-discordant regions included key genomic regulators of lineage plasticity, osteomimicry, and cellular differentiation, including $M Y C N$ gain in CTCs (31\%) that was rarely detected in cfDNA. CTC MYCN gain was associated with poor clinical outcomes in AR-V7 negative men and small cell transformation. In conclusion, we demonstrated concordance of multiple genomic alterations across CTC and cfDNA platforms; however, some genomic alterations displayed substantial discordance between CTC DNA and cfDNA despite the use of identical copy number analysis methods, suggesting tumor heterogeneity and divergent evolution associated with poor clinical outcomes.
\end{abstract}

This article is protected by copyright. All rights reserved. 


\section{Introduction}

Circulating tumor cells (CTCs) are rare cancer cells that are shed in the peripheral bloodstream during metastatic dissemination and progression [1, 2]. Previous studies have suggested CTC enumeration could be useful for prognosis and clinical response assessments for patients with advanced cancer while providing valuable information for drug target identification, treatment monitoring, and exploring tumor heterogeneity [3-6]. Tumor-derived cell-free DNA (cfDNA) in serum or plasma likely originates from apoptotic, dead or dying tumor cells, and may constitute genetic information associated with tumor cell evolution and heterogeneity during treatment and metastatic progression [7,8]. Despite the utility of CTC and cfDNA analyses, there are several major challenges inherent in the development of CTC or cfDNA biomarkers [9] for treatment selection in clinical studies, namely reproducibility, defining actionable targets, and consistency across platforms [10, 11]. While some studies have found concordance between selected cfDNA genomic alterations and paired tumor biopsies $[12,13]$, few studies have examined the consistency of wholegenome alterations across paired CTC and cfDNA samples.

Metastatic castration resistant prostate cancer (mCRPC) is a lethal and complex disease [6]. While most men initially respond to novel hormonal therapies, such as abiraterone acetate or enzalutamide, nearly all men with $\mathrm{mCRPC}$ relapse and develop resistant progression over 1-3 years [14]. In addition, cross-resistance between abiraterone and enzalutamide is common in $>50 \%$ of patients who are 
treated sequentially [15]. Thus, optimal delivery of these agents in the second-line setting could be facilitated by the development of predictive biomarkers of treatment response and resistance. For example, detection of AR-V7 in CTCs, an important splice variant in the androgen receptor, is associated with resistance to hormonal therapies abiraterone acetate and enzalutamide [16-19]. Yet, despite the importance of AR-V7 as a predictive biomarker, many men with AR-V7 negative disease have resistance to therapy or develop resistance over time that is currently unexplained by $A R$ alterations $[16,17,20]$. Thus, novel approaches to detect de novo biomarkers are needed. CTC- and cfDNA-based methods are minimally invasive technologies that can provide longitudinal insight into tumor biology of individual patients and provide a source of biomarkers for MCRPC response and progression.

Given the increasing number of systemic treatment options available for men with mCRPC, predictive biomarkers that report on the underlying tumor biology and potential drug sensitivity would be desirable to maximize benefit and minimize harm and cost $[21,22]$. However, metastatic biopsies in men with mCRPC are challenging, invasive, may not result in enough metastatic tissue for analysis, and can be limited by tumor heterogeneity or plasticity [22]. Conversely, CTC DNA and cfDNA are a ready source of cancer cell DNA that can be noninvasively collected and profiled longitudinally for molecular analysis for association with outcomes and potential mechanisms of drug resistance $[23,24]$. However, the concordance of genomic alterations between CTC DNA vs. cfDNA is not well established, and data reproducibility and concordance across similar cfDNA platforms has proven to be a major challenge $[12,25]$. Further, CTCs and cfDNA may represent different sources of tumor tissue and, in theory, could provide unique readouts of cancer biology and tumor heterogeneity over time depending on the specific clinical context and biomarker.

Recently, studies have characterized whole-genomic copy number alterations (CNA) in CTCs and cfDNA; however, no systematic assessments of paired CTC and cfDNA 
exist, especially in men with mCRPC [8, 10, 12, 26-29]. To address this, we analyzed whole-genome CNA in paired CTCs and cfDNA from men with mCRPC. Here, we describe the frequency of chromosomal aberrations in men with MCRPC treated with abiraterone, enzalutamide, or radium-223 and focus on genomic regions with concordance and discordance in paired CTCs and cfDNA and their association with clinical outcomes. The frequency and reproducibility of CTC-discordant CNAs were confirmed in publicly- available clinical samples, where both DNA CNA and mRNA expression were available. Our findings suggest that CTCs may provide unique biologic insights into MCRPC heterogeneity and clinical outcomes that may be missed through cfDNA analysis alone.

\section{Materials and Methods}

\section{Patient selection}

We included two independent prospective cohorts of men with MCRPC, including men treated with radium-223 (NCT02204943) or abiraterone/enzalutamide (PROPHECY study [30], NCT02269982). All patients at study entry had mCRPC and progressive disease and were histologically confirmed to have prostate adenocarcinoma, evidence of metastases by clinical/radiographic, castrate levels of testosterone ( $\leq 50 \mathrm{ng} / \mathrm{dl}$ ), and evidence of cancer progression by computerized tomography/bone scan or prostate-specific antigen rises by The Prostate Cancer Clinical Trials Working Group (PCWG2) criteria [31]. PROPHECY patients were required to have two poor-risk features, including anemia, high $\mathrm{LDH}$, high alkaline phosphatase, pain requiring opiates, PSA doubling time of $<3$ months, radiographic 
progression, and prior abiraterone/enzalutamide therapy with planned cross-over to the other hormonal agent. For the radium-223 study, patients were excluded if they had visceral metastases, and men were eligible if they had more than two symptomatic bone metastases. All patients provided informed consent under separate protocols approved by the Duke University Institutional Review Board (IRB, PROPHECY, and Radium-223) or the Weill Cornell Medical College IRB (PROPHECY).

\section{CTC DNA and cfDNA isolation}

CTCs were enumerated using the FDA-approved CellSearch platform in a Clinical Laboratory Improvement Amendments (CLIA)-approved laboratory [11]. Separately, for CTC isolation for copy number alteration (CNA) and aCGH, 7.5ml blood in EDTA tubes was diluted with blood lysis buffer (Gibco, A10492), centrifuged and washed with $0.5 \%$ BSA in PBS buffer, and incubated for 30 minutes at $4 C^{\circ}$ with Dyna BeadsCD45 (Invitrogen, 11153D) to deplete leukocytes. The enriched cells were stained with an anti-EpCAM antibody (Serotek, AbD, CMA1870g) labeled with anti-Mouse IgG1 AlexaFluor-647 (Z25008, Invitrogen) and anti-Hu CD45 coupled to AlexaFluor488 (C1620, Leinco). Next, EpCAM+ and CD45- cells were sorted by flow cytometry [32]. Gating thresholds were set using unspiked and spiked EpCAM+ (T47D) cells into healthy volunteer blood. In parallel, peripheral blood mononuclear cells (PBMCS) were isolated from the blood collected in EDTA tubes for germline DNA analysis as controls for each CTC sample, diluted with PBS (1:1), and layered on 4ml of FicollPlaque (GE Healthcare, 17-1440). Ficoll-Plaque layers were washed and suspended in $100 \mu \mathrm{l}$ in Milli-Q-Water. For cfDNA isolation for CNA and $\mathrm{aCGH}, 2 \mathrm{ml}$ plasma was obtained from mCRPC patients and isolated using the QIAamp Circulating Nucleic Acid Kit (Cat No-55114, Qiagen) and quantified using Quant-iT TM PicoGreen ${ }^{\text {TM }}$ dsDNA Assay Kit (Thermo Fisher Scientific, Cat no: P11496) by Tecan Infinite ${ }^{\circledR} 200$ PRO. CfDNA visualized on an Agilent 4200 TapeStation. Before hybridizing the 
samples to the aCGH microarray, all DNAs were amplified by using the GenomePlex® Single Cell Whole Genome Amplification Kits (Sigma, WGA4) to the yield required for labeling $(0.50 \mu \mathrm{g} / \mathrm{sample})$ according to Agilent's aCGH manual. Amplified genomic DNA was visualized on 1.5\% agarose gel to verify DNA 200700bp (Figure S1).

\section{AR-V7 testing}

The Epic AR-V7 nuclear protein detection and JHU AR-V7 RNA based assays were used to detect AR-V7 in CTCs. A positive test for the Epic assay was defined as the presence of CTCs ( $\mathrm{CK}+/-)$ expressing nuclear AR-V7 protein, as previously described [19, 33, 34]. The JHU AR-V7 assay is an RT-PCR based assay to detect AR-V7 mRNA expression [16, 30].

\section{Array comparative genomic hybridization (CGH)}

Two-color probe-based aCGH was performed using Agilent Oligonucleotide Arraybased CGH for Genomic DNA Analysis (Sure Print G3 Human CGH array, 4x180K) [32]. Data were analyzed using the Agilent Cytogenomics Software, and all CNAs were manually analyzed [32]. CNAs were then cross-referenced with publiclyavailable prostate cancer datasets using cBioportal [35]. The biological or clonality of CTCs genomic concordance (CNA present in CTCs were detected in cfDNA) and discordance (CNA present in CTCs, but not detected in paired cfDNA samples) were calculated by comparing CNA in CTCs and cfDNA. Next, to minimize the false positives result, stringent filtering criteria were applied by using a minimum 3+ contiguous probes distribution, and two independent calls were used to call a copy gain or loss event. Further, all genomic altered genes were assessed manually based on aCGH probe distribution within chromosomal aberrations region analyzed in the Agilent CytoGenomics software. In addition, genomic agreement and 
disagreement between 64 CTCs and paired cfDNA CNAs (gain versus no gain, or loss versus no loss) from combined radium and PROPHECY studies were independently analyzed by Cohen's Kappa method in Graph Pad prism software (Table S3).

\section{Low-pass whole genome sequencing, tumor content and copy number analysis in cfDNA}

Low-pass whole genome sequencing of 83 cfDNA from 73 mCRPC patients was generated using ThruPLEX DNA-seq kits according to the manufacturer's protocol with the Ion Torrent IonXpress barcode index and sequencing adapters. Libraries were quantified using an Agilent Bioanalyzer 2200 tape station with HS D1000 tape and sequenced with 16 samples per Proton PI chip on an Ion Proton sequencer. To determine the tumor content (TC) in cfDNA, we used a well-established method PRINCe (pan-cancer, rapid, inexpensive, whole-genome NGS of cfDNA approach) for tumor content determination and to identify focal CNAs in cfDNA from mCRPC patients' samples via low coverage ( 0.01x) through genome-wide CNAs analysis, where the least-squares based distance metric (LSS) was performed on wholegenome copy number data, and guide the tumor content approximation with low tumor content samples (LSS < 0.1), where estimated tumor contents greater than $8.75 \%$ by LSS analysis were considered as high tumor content as previously described by Daniel H. Hovelson et al., 2017 [36]. Focal CNAs were measured as CNAs 1.5-20 Mb long with the log2CNRatio cutoffs for genomic gain and loss analyzed in IpWGS were $>=0.50$ and $<=-0.50$, respectively.

\section{Clinical Outcomes and Statistical Analysis}

Genomic CNA data from 40 baseline samples were grouped as copy gain, loss or neutral, and investigated for the presence of CNA and their associations with progression-free survival (PFS) with abiraterone/enzalutamide in the PROPHECY 
study. Progression-free survival was defined as the date from registration to radiographic progression using PCWG2 criteria (26), clinical progression requiring a change in systemic therapy, or death, whichever came first. Kaplan-Meier analysis was used to estimate the PFS distribution according to the presence of CTC DNA or cfDNA specific genomic alterations in men who were AR-V7 negative as defined by the Epic AR-V7 nuclear assay. The proportional hazards model was utilized to estimate the hazard ratios for PFS in men who were AR-V7 negative. Besides, Graph pad prism was used for plotting the graphs, and p-value calculation based on two-tailed Mann Whitney test. 


\section{Results}

\section{Patients with mCRPC demonstrate heterogeneous cfDNA concentrations that correlate with CTC burden}

A total of 140 men with progressive MCRPC were consented and enrolled in two independent prospective studies prior to initiating a new systemic therapy. Patients were treated with enzalutamide or abiraterone acetate targeting androgen receptor signaling ( $n=120$, PROPHECY clinical trial, NCT02269982) [19] or radium-223 ( $n=20$, radium-223 pharmacodynamic trial, NCT02204943) [37] targeting bone metastasis. As shown in Figure 1A and B (CONSORT diagrams), 93 of these men contributed either CTCs, cfDNA, or both in this analysis of the combined studies. Together, 32 patients contributed 64 time-matched/paired samples of both CTCs and cfDNA for the present concordance analysis. In the PROPHECY study, men had a median age of 73 years (range 45-92), median PSA of 19ng/ml (0.08-4194); mean 123, high alkaline phosphatase, prior abiraterone/enzalutamide therapy or radiographic progression, and 100\% had bone metastases. In the radium-223 prospective study, patients with mCRPC were enrolled with $>2$ symptomatic bone metastases. The median age was 72 years (range 54-86), median PSA was 50 $\mathrm{ng} / \mathrm{ml}$ (range 2-1896), and all patients had elevated serum alkaline phosphatase. A total of $95 \%$ of patients in the radium-223 study had prior enzalutamide, and $80 \%$ had prior abiraterone acetate/prednisone treatment. Tables S1-2 describe the baseline clinical characteristics of the radium-223 and PROPHECY cohorts of men with MCRPC, respectively. Figure 1 provides the CONSORT diagram for the patients included in the present analysis and describes the research plan in the combined cohort of 140 mCRPC men. A total of 52\% (69/133) of mCRPC men at baseline had an unfavorable $\geq 5$ CTCs per $7.5 \mathrm{~mL}$ blood by CellSearch criteria [6], 
the median number of CTCs was 5 (Figure S2A), and 30 men had 0 CTCs detected by CellSearch criteria.

Similar to CTC counts, we found a range of cfDNA concentrations (median 104 $\mathrm{ng} / 2 \mathrm{ml}$, range 46-1,458 ng/2ml plasma) across all patients. In addition, unamplified cfDNA was visualized on Agilent TapeStation revealed two DNA bands between 100-200bp and 500-700bp, where, no genomic DNA contamination was seen (Figure S3). We hypothesized that this variation in cfDNA concentration could be explained, at least in part, by the CTC count. We hypothesized that men with higher CTC burden would also have higher cfDNA concentrations [11]. To test this hypothesis, we examined the association of cfDNA concentrations with CTC enumeration by measuring cfDNA concentration among 90 mCRPC men. Consistent with our hypothesis, we found that the median cfDNA concentration was higher in the group of patients with $\geq 5$ CellSearch CTCs (median $118 \mathrm{ng} / 2 \mathrm{ml}$ ) in comparison to those men with $\leq 4$ CTC $(\mathrm{p}$-value $=0.003)($ median $85 \mathrm{ng} / 2 \mathrm{ml})($ Figure S2B$)$.

\section{Low-pass whole genome sequencing, an alternative approach for copy number and tumor content determination in mCRPC}

We next sought to evaluate tumor content CNAs in cfDNA. To do this, we first utilized a low-pass whole-genome sequencing method (IpWGS, coverage $\sim 0.1 \mathrm{x}$ ), which requires an input of cfDNA (1-5ng). We applied this technique to cfDNA isolated from 83 plasma samples from 73 men in the PROPHECY study (72 baseline and 11 progression) [19]. The tumor content was determined in cfDNA from wholegenome sequencing data using the PRINCe method according to previously described methods [36]. After determining the tumor content in cfDNA, we compared the distribution of CellSearch CTCs in 68 baseline mCRPC men from PROPHECY study with low tumor content (lowTC) $(n=40)$ and high tumor content (highTC) $(n=28)$. We found that CellSearch CTC counts were significantly higher in highTC 
cfDNA ( $p$ - value= 0.0001) in comparison to lowTC cfDNA samples (Figure S2C). While no significant differences in cfDNA concentration were noted in high vs. low TC samples (Figure S2D). Furthermore, to prove the hypothesis that men with higher CTC burden would also have higher tumor content, here, we examined the association of tumor content (lowTC vs. highTC) with CTC enumeration (0, 1-4 and $>=5$ CTC CellSearch) among 68 mCRPC men, and found that 71\% (20/28) patients with highTC had >=5 CellSearch CTCs in comparison to those men with $\leq 4$ CTC (Figure S2E). Finally, these data suggest that men with high CTCs tend to have a greater cfDNA tumor content.

Using IpWGS, we detected the androgen receptor (AR) copy gain in 36\% (30/83) of cfDNA samples. Of these, 70\% (21/30) of patients with AR copy gain had highTC, while 30\% (9/30) had lowTC in their paired cfDNA (File S1). Next, we compared cfDNA CNA reproducibility between aCGH and IpWGS by comparing whole genome and focal/gene-level copy number changes at the $A R$ locus in 21 paired cfDNA samples from 15 mCRPC men (15 baseline and 6 progression) (Figure 1B). We identified high concordance (genomic alteration identified by both aCGH and IpWGS) of both AR copy gain detection (89\%) and AR copy neutral status (92\%) (Figure S4A-D). In highTC samples, AR gain detection was 67\%, while in lowTC samples $A R$ gain detection was only $33 \%$, suggesting that TC clearly impacted the ability to detect this genomic alteration. The analysis of $A R$ copy numbers provided an estimate of data reproducibility of cfDNA profiles across different whole-genome platforms.

CTC DNA vs. cfDNA: whole-genome copy number alteration analysis by aCGH After establishing the impact of TC on the detection of common genomic alteration in cfDNA using both low pass WGS and aCGH, we next compared CNAs in patients' paired CTC DNA and cfDNA by identical aCGH methods across both the radium-223 and PROPHECY prospective studies. For this analysis, we included 69 CTC DNA from 35 men (25 pre-treatment baseline samples and 40 longitudinal samples) and 
72 paired cfDNA from 34 men (33 pre-treatment baseline and 39 longitudinal) with paired germline reference DNA (Figure 1A and B). In this comprehensive analysis, we focused on sixty common and recurrently altered genes that have been previously implicated with mCRPC for CNAs analysis in both CTCs and cfDNA [38]. We identified heterogeneity in the detection of multiple altered genomic regions in CTCs- and paired cfDNA samples, including common genomic gains in FOXA1 (59\% vs. $75 \%$ ), KDM6A (52\% vs. $18 \%$ ), AR (46\% vs. $63 \%$ ), and MYC (17\% vs. $21 \%)$ (Figure 2A). Similarly, common genomic losses included ZFHX3 (59\% vs. 40\%), FGFR2 (43\% vs. $33 \%$ ), PHLPP1 (36\% vs. $29 \%$ ), BRCA1 (26\% vs. $28 \%$ ), and PTEN (14\% vs. 13\%) (Figure 2B). For example, at a focal or gene level, a representative gene view image of concordance in CTCs vs. cfDNA harboring AR copy gain and ZFHX3 copy loss with probes distributions are shown in Figure $\mathbf{2 C}$ and $\mathbf{D}$, respectively.

Further, we compared the prevalence of these CNAs from our cohort with publiclyavailable datasets in cBioportal [35]. The prevalence of these genomic alterations in both CTCs and cfDNA was similar to those reported in multiple primary and metastatic prostate cancer datasets, with copy gain or loss in the same direction as those identified in CTCs and cfDNA. For example, 52\% (78/150) of SU2C/PCF mCRPC samples had $A R$ gain, which is consistent with our $A R$ copy gain detection in CTCs DNA and cfDNA (46\% vs. $63 \%$ ) (Figure 2E). In addition to $A R$, the prevalence of gain or loss of multiple additional genomic regions was confirmed in publicly-available prostate cancer data sets, including amplification of FOXA1, CYP11B1, and MYCN (Figure 2E), and deletions in ZFHX3, NCOR1, FGFR2, and NKX3-1 (Figure 2F). Thus, our findings suggest that aCGH consistently detects concordant CNAs in CTCs and cfDNA from men with mCRPC, similar to that reported from metastatic biopsies.

To examine whether there are discordant genomic alterations between time- and patient-paired CTC DNA and cfDNA, we first analyzed the prevalence of genomic 
alterations between 32-paired CTCs and cfDNA from 16 mCRPC men from the radium-223 treated cohort of men with MCRPC, including 14 baseline and 18 longitudinal samples. We observed a high concordance in copy number gain of FOXA1 (86\%) and AR (87\%) between matched CTC and cfDNA sources, moderate concordance for copy gain of MYC (50\%), NCOR2 (50\%), AURKA (50\%) and loss of ZFHX3 (47\%) and PHLPP1 (45\%), and low concordance between sources for gain of MYCN (11\%), KDM6A (39\%) and loss of BRCA1 (30\%), RB1 (33\%), and FGFR2 (29\%) in CTC and matched cfDNA (Figure S5A). For example, MYCN gain was detected in $28 \%$ of CTC DNA samples but was detected in only $9 \%$ of cfDNA samples. These data suggest significant variability in the detection of genomic alterations using these two different sources of tumor DNA.

We next examined the degree of CNA discordance within each treatment cohort, where tumor heterogeneity was observed in both CTC and paired cfDNA. A number of discordances were observed between 32 paired CTCs and cfDNA (14 baseline and 18 progression) from the 16 men with mCRPC treated with radium-223, such as gain of CYP11B1 detection rate (59\% vs. $3 \%$ in CTCs vs. cfDNA, respectively), and MYCN (28\% vs. 9\%), and copy loss in GRHL2 (75\% vs. 19\%), RUNX2 (63\% vs. $13 \%), P X N(38 \%$ vs. 0\%), and NKX3-1(19\% vs. 6\%) (Figure 3A). These discordances were observed despite clear probe coverage of the genomic regions of interest in paired CTCs and cfDNA, as illustrated in Figure S6A and B. In addition, these discordant gain or loss of genomic regions were also observed in publicly available prostate cancer tissues data sets, as shown in Figure S7.

To externally validate these CTC-discordant genomic alterations, we analyzed CTC vs. cfDNA concordance in the multicenter prospective PROPHECY study of men with MCRPC treated with abiraterone or enzalutamide, including 32 paired cases with matched CTCs and cfDNA samples from 16 mCRPC men (15 baseline and 17 progression) treated with abiraterone or enzalutamide. Similar to our radium-223 treated cohort, we detected concordant gains in FOXA1 (100\%), AR (40\%), MYC 
(40\%), and loss of ZFHX3 (58\%), PHLPP1 (50\%), BRCA1 (50\%), RB1 (40\%), and FGFR2 (50\%) between paired CTC and cfDNA sources (Figure S5B).

In the PROPHECY cohort, we likewise observed discordant gains in MYCN (detection frequency of 31\% vs. 0\%) and CYP11B1 (34\% vs. 9\%), and discordant loss in RUNX2 (47\% vs. 3\%) and PXN (28\% vs. 3\%) (Figure 3B), respectively. We next asked whether this CTC-discordance could be due to lowTC or due to high CTC counts in the paired samples. Surprisingly, however, we found similar rates of high CTC vs. cfDNA discordance even in those cfDNA cases with highTC or low CTCs (Figure 3C and D). Moreover, the prevalence of genomic alterations of these concordance/discordances were significantly higher in > =5 CTCs in comparison to 0 , and 1-4 CTCs by CellSearch criteria (N=45 baseline, PROPHECY study) (Figure 3E). In addition, we compared the overall genomic alterations, and genome discordance in CNAs at the whole-genome level in 21 paired CTCs and cfDNA based on their tumor content grouped into lowTC $(n=12)$ and highTC $(n=9)$. We observed a trend of increased in overall CNAs (Figure S8A), and genomic discordance of genomic regions with CTC DNA copy gain in highTC than lowTC samples, but no significant differences were observed in genomic regions with cfDNA copy gain or loss (Figure 3F-H, and Figure S8B and C). Further, to visualize inter- and intra-tumor heterogeneity among these CNAs in detail, the number of CTCs, and paired CTCs vs. cfDNA CNAs status of 60 genes from radium (top) and PROPHECY (bottom) studies are summarized in Figure S9A and B. Analysis of genomic copy gain agreement by Cohen's Kappa in 64 paired CTCs and cfDNA CNAs was consistent with most of our previous concordance and discordance findings. However, we found low Cohen's Kappa for $A R$ gain, reflecting more abundance of $A R$ gain detection in cfDNA over CTC DNA. Analysis of genomic copy Ioss agreement by Cohen's Kappa, however, was variably consistent with our previous findings. The top agreement and disagreement between CTCs and cfDNA CNAs with Kappa scores are summarized in Table S3. Together, these data suggest that CTCs in some men with mCRPC have consistently different copy number 
profiles as compared with paired cfDNA profiles, as exemplified by selected gene regions, which may be reflective of either biologic heterogeneity of the source of DNA or differences in detection for each assay related to CTC number or cfDNA concentration.

\section{Genomic and phenotypic evolution: heterogeneity and NEPC transformation}

To describe in detail the potential clinical significance of discordant CTC and cfDNA profiles, we collected paired CTCs and cfDNA with high tumor content from a PROPHECY cohort patient (\# 809-33) at baseline and disease progression during treatment with abiraterone and enzalutamide. The patient had been determined to be negative for AR-V7 by the Epic AR-V7 assay [17] and had a CTC aCGH profile consistent with gains in AR, MYCN, FOXA1, and loss of BRCA1. His CTC phenotype suggested high tumor heterogeneity with an elevated Epic Shannon Index of 1.7 [39]. Following continued disease progression on enzalutamide and docetaxel chemotherapy, the patient's CTCs increased from 194 to 1,359, and his cfDNA concentration increased from 129 to $428 \mathrm{ng} / 2 \mathrm{ml}$. These increases in cfDNA were accompanied by a rapid PSA rise and the development of soft tissue and liver metastases. A metastatic biopsy of a lymph node confirmed histologic transformation to small cell carcinoma (Figure 4). His repeat CTC genomic analyses remained negative for AR-V7, but demonstrated an aCGH profile consistent with $A R$ genomic loss, the persistence of MYCN gain and a small cell CTC and tissue phenotype suggestive of a neuroendocrine-like transformation [40] (Figure 4A). Interestingly, MYCN copy gain was found in longitudinal CTC samples but not in cfDNA despite the highTC in his cfDNA collected from the same time point. In addition, several new genomic alterations became dominant in this patient's post-enzalutamide progression CTCs samples that were not detected in matched cfDNA samples (Figure 4B-D). The patient's tumor cells also exhibited high nuclear to cytoplasmic ratio, inconspicuous nucleoli, foci of necrosis and abundant apoptotic debris, and PSA loss of expression, all of which are features characteristic for small cell 
carcinoma. Immunohistochemical staining of pan-cytokeratin and synaptophysin confirmed the neuroendocrine differentiation of an epithelial lineage in this tumor while highlighting the minimal cytoplasm present in these tumor cells (Figure 4E-H).

To assess the relevance of these findings in a broader context, we established that MYCN gain is one of the top discordant CNAs in PROPHECY abiraterone/enzalutamide treated mCRPC men (31\% CTC vs. 0\% cfDNA) (Figure 4la). In support of the PROPHECY findings, MYCN gain was also observed to be discordant in 32 matched CTCs and cfDNA from 16 mCRPC men treated with radium-223 (28\% MYCN gain detection in CTC vs. 9\% in matched cfDNA) (Figure 4lb). In addition, MYCN gain was observed in multiple publicly-available prostate cancer datasets, including both primary and metastatic tumors (TCGA, SU2C, and NEPC) in cBioportal [35] (Figure 4lc). Taken together, these data suggest that CTCs may discern clonal selection of $A R$-independent and treatment-emergent genomic events that may be contributing to enzalutamide resistance and may not be identified in cfDNA.

\section{Genomic discordance of osteomimicry genes in MCRPC}

Among those genes with discordant gains or losses in our MCRPC cohort (Figure $3 A$ and $B$ ) were genes related to osteoblast biology, suggesting a role for potential osteomimicry in metastatic dissemination in men with mCRPC [37, 41]. We identified discordant gains and losses in seven key osteoblastic regulating genes: $A L P L$ (alkaline phosphatase), RUNX2 (runt-related transcription factor 2), BGLAP (bone gamma carboxyglutamate protein, osteocalcin), SPP1 (secreted phosphoprotein 1, osteopontin), CDH11 (osteoblast cadherin), TNFSF11 (tumor necrosis factor ligand superfamily 11 or RANKL) and SPARC (secreted protein acidic and cysteine-rich, osteonectin). When examined by aCGH in 32 paired CTCs and cfDNA DNA from 16 mCRPC men, we found genomic gains in CTCs for ALPL (12\%), CDH11 (19\%), SPP1 (22\%), and TNFSF11 (13\%), whereas copy loss of RUNX2 was notable in $63 \%$ of CTCs. Of these, $69 \%$ of RUNX2 copy loss cases also harbored highTC in 
their matched cfDNA samples. Remarkably, however, we did not identify common gains or losses of these osteoblastic gene regulators in cfDNA samples by aCGH from the same patients at the same time points (Figure S10A). It is important to note that gains and losses at other loci were highly concordant in these same-paired samples using the same aCGH methodology, suggesting that differences in osteoblastic gene alterations found in CTCs and absent cfDNA were not due to differences in CTC number, cfDNA concentration, or assay sensitivity. For example, we found common genomic gains in AR and FOXA1, and losses in PHLPP1 and BRCA1 in both CTCs and matched cfDNA from the same patients at the same time points, and while slight differences were noted in the frequency of these gains in the cfDNA, there was overall consistency between CTCs and cfDNA of these genes.

We also observed discordant genomic findings of osteoblastic regulators in CTCs in our PROPHECY cohort of 32 CTCs and matched cfDNA from 16 patients. For example, we identified copy gains for these seven osteoblast genes in patients, including ALPL (19\%), CDH11 (16\%), SPP1 (9\%), and TNFSF11 (6\%) and SPARC (9\%), whereas, copy loss of RUNX2 was notable in 47\% of CTCs [19]. However, in matched cfDNA samples from the same patients, copy gain/loss of these key genes were not observed despite the collection of samples at the same time points and using the same aCGH method of detection, and despite highTC of many cfDNA samples (Figure S10B). In detail, the CNAs in osteomimicry genes in paired CTCs and cfDNA from radium (top) and PROPHECY (bottom) studies are summarized in File S2. These data, together with discordances of MYCN gain in CTCs, suggest that CTCs may harbor genomic alterations important to the metastatic biology of CRPC that is missed by examining only the cfDNA fraction.

We next sought to validate the presence of key genomic alterations identified in our CTCs, particularly those discordant genes not found in cfDNA, in public datasets of men with mCRPC. To explain if genomic alteration confers mRNA expression in mCRPC, we analyzed two independent prostate cancer datasets containing 286 
metastatic tissue samples [38, 42], where both CNV and mRNA-expression profiling were performed simultaneously and analyzed in cBioportal [35]. We identified several CTC-discordant genes, such as KDM6A, UGT2B17, NRAS, and NCOR1, in which genomic loss correlated with lower mRNA expression and genomic gain correlated with higher mRNA expression in mCRPC tumor tissues (Figure S11). However, for several CTC discordant genes, we failed to identify a relationship between these CNAs and altered mRNA expression and CNAs. These data support our findings that several CTC-specific genomic alterations may be a common driving mechanism in $\mathrm{MCRPC}$, but caution that some genomic alterations may not be associated with mRNA dysregulation or have functional significance, and thus require mechanistic and functional validation.

\section{Genomic loss of PTEN is prognostic for poorer survival in AR-V7 negative patients}

Finally, we sought to examine the clinical implications of CTC-specific genomic alterations in our PROPHECY study cohort. We focused our analyses on those men with MCRPC who tested negative for AR-V7 nuclear protein using the Epic AR-V7 test $[30,33]$, given that these men have significantly heterogeneous clinical outcomes when treated with abiraterone or enzalutamide. First, as a positive control biomarker, we examined the prognostic relevance of AR-V7 mRNA detection by the Johns Hopkins Adnatest method (JHU AR-V7) in CTCs of 40 baseline mCRPC men in the PROPHECY study who tested negative for AR-V7 by the Epic nuclear assay (Epic AR-V7) [16, 17]. A total of 18\% (7/40) baseline CTCs samples were AR-V7 mRNA positive (median CTC 45, range 3-194) by the JHU AR-V7 assay, but negative by Epic AR-V7 nuclear protein detection assay. The median radiographic PFS with abiraterone or enzalutamide treatment was significantly lower at 2.7 months for AR-V7 positive disease as compared with 8.4 months for AR-V7-negative disease (HR 3.2, 95\% Cl, 1.4 - 7.4) (Figure 5A). These data support the relevance of JHU CTC AR-V7 positivity, even in Epic AR-V7 negative men. 
Next, we examined common concordant CNAs in CTCs for their prognostic relevance in 40 Epic AR-V7 negative men with mCRPC treated with abiraterone/enzalutamide. A total of $45 \%$ (18/40) cases harbored $A R$ copy gain by aCGH. These patients with AR copy gain had a trend toward lower median PFS (4.7 months) vs. copy neutral/loss (8.4 months, HR 1.6, 95\% Cl, 0.8 - 3.2) (Figure 5B), though these differences were not statistically significant. In addition, the median PFS of men with CTC PTEN loss was significantly lower at 3.4 months in comparison to 8.4 months for men without PTEN loss (HR $0.4,95 \% \mathrm{Cl}, 0.2-0.8$ ) (Figure 5C). Given the small sample size, we are unable to perform multivariableadjusted analyses including CTC enumeration or other clinical prognostic factors, and these results are hypothesis-generating only.

We next explored the prognostic potential of common CTC-cfDNA discordant genes and their association with PFS in these 40 Epic AR-V7-negative men with mCRPC treated with abiraterone or enzalutamide. Patients with CTCs who had discordant copy gains in MYCN, BRCA2 or KDM5D, or had discordant copy loss of RUNX2 had shorter progression-free survival (PFS) times compared with patients whose CTCS were copy neutral for these genes. For example, median radiographic PFS was 3.5 months for MYCN copy gain in CTCs compared with 8.4 months for copy neutral $(\mathrm{HR}=1.9,95 \% \mathrm{Cl}, 0.9$ - 3.9) (Figure 5D). Similarly, for BRCA2, PFS was significantly shorter at 2.8 months for copy gain and 8.4 months for copy neutral $(\mathrm{HR}=4.3,95 \% \mathrm{Cl}, 1.9$ - 9.8) (Figure 5E), whereas PFS for RUNX2 copy loss was 3.8 months compared with 7.4 months for copy neutral $(\mathrm{HR}=0.8,95 \% \mathrm{Cl}, 0.4-1.6)$ (Figure 5F). The median PFS for additional concordant and discordant copy gains or losses are summarized in Figure S12A-F. Moreover, some gains/losses were not associated with outcomes, suggesting that just detection of an alteration as a surrogate of high CTC number is insufficient for prognostication, and those specific genomic alterations are likely critical. While we consider these results exploratory and hypothesis-generating, our analyses suggest the following: 1) both concordant 
and discordant CNAs in CTCs versus cfDNA may be clinically relevant and prognostic for outcomes with AR inhibitor therapy; 2) CTC genomic alterations provide a unique source of DNA (and RNA) as compared to cfDNA, and 3) these differences may impact metastatic biology and the progression of treatment resistance.

\section{Discussion}

In this study of prospectively-collected blood samples from two independent cohorts of men with mCRPC, we identified critical discordances in a series of genes related to prostate cancer pathobiology in CTC DNA compared to patient- and time-paired cfDNA samples. Our study confirms that the detection of whole-genome alterations is feasible in the majority of patients, and we identified common concordantly-altered genes in CTCs and cfDNA, such as AR, FOXA1, and PTEN. We identified strong concordance of $A R$ genomic alterations between paired cfDNA samples using both aCGH and IpWGS in cases with high and low tumor content. Interestingly, we found consistent discordances in CTC samples, which were identified in independent cohorts, even in the presence of high cfDNA tumor content, suggesting that the genomic copy discrepancies identified between CTC DNA and cfDNA sources are platform-independent and may suggest different biological processes. While detection of genomic alterations is challenging in the absence of CTCs or lowTC plasma samples, these data support divergent biology in CTCs that may have clinical implications for therapy and biomarker development. 
We observed significant between-patient and longitudinal genomic heterogeneity of CTCs and cfDNA in mCRPC patients, suggesting the presence of patient-specific diversity as well as clonal evolution over time. The detection of CTC-specific genomic alterations was linked to 1) neuroendocrine and lineage plasticity regulators, such as MYCN gain, 2) osteomimicry regulators, such as RUNX2 loss, and 3) epigenetic regulators, such as KDM6A and KDM5D. These CTC-specific alterations were associated with shorter progression-free survival times with abiraterone or enzalutamide in AR-V7 negative men with $\mathrm{mCRPC}$, suggesting that CTCs may harbor clinically-relevant genomic information that could be missed by focusing solely on cfDNA analysis. Critically, these poor outcomes were present in AR-V7 negative men, an important group of patients with a wide range of clinical outcomes with AR inhibitor therapy. We identified variable concordance in CTC DNA vs. cfDNA CNAs depending on the specific gene, type of alteration, and CTC enumeration, possibly reflecting biologic variability and single-cell tumor heterogeneity.

We also established that aCGH and IpWGS were highly concordant in detecting $A R$ (89\%) gain in the same cfDNA samples. We also showed in MCRPC that CTCs and cfDNA are largely concordant in key genes, likely reflecting the clonal origin of CTCs [43]. For example, $A R$ and FOXA1 gains were commonly observed in both CTC and cfDNA compartments and mirrors that of public mCRPC datasets. In a prospective study, Wyatt et al. (2017) compared genomic alterations in paired metastatic prostate cancer tissues and cfDNA using whole-exome sequencing and identified $64.7 \%$ concordance in $A R$ amplification detection [12]. Similarly, we also confirmed $A R$ copy gain concordance between paired CTC DNA and cfDNA samples collected from the same patients and at the same time point from two-combined concordance across both datasets, and in highTC samples. The amplification of $A R$ in baseline CTC DNA was also associated with shorter survival times. Hence, this aCGH assay is appropriate to reproducibly identify alterations of selected driver genes, such as 
$A R$, which supports the development of liquid biopsy platforms using CTC or cfDNAbased biomarkers to understanding potential resistance mechanisms in cancers at the molecular level [38, 44].

Interestingly, we also observed a number of discordant genomic alterations that may be critical to tumor progression, drug resistance, and lethal disease heterogeneity [43]. For example, previous studies have shown that MYCN is amplified and capable of inducing a neuroendocrine-like prostate cancer phenotype, where it is thought to be involved in developing resistance to androgen deprivation therapy $[45,46]$. In our PROPHECY study, MYCN copy gain was observed in 31\% of CTCs from mCRPC, whereas, none of the paired cfDNA cases harbored copy gain. The presence of MYCN in CTCs was also associated with shorter PFS with abiraterone or enzalutamide treatment, which suggests that CTC DNA and not cfDNA, may be more useful biospecimen source of certain prognostic or predictive biomarkers, and supports the enrichment of NEPC-like CTCs and non-AR dependent mCRPC during hormonal therapy [47]. The lack of $M Y C N$ gain in cfDNA may relate to the fact that CTCs remain viable in the circulation and are not shedding sufficient DNA to be detected in the cfDNA assays. More sensitive cfDNA assays may reduce this discordance with CTC DNA detection; however, our study illustrates the need for close attention to both assay sensitivity and biologic heterogeneity.

Our observation of discordance in genes detected in CTC CNAs that regulate osteoblast biology suggests the importance of these genes to bone metastasis. One hundred percent of the men in our two cohorts had bone metastases, and we identified genomic alterations in seven osteomimicry markers in mCRPC CTC and paired cfDNA from two independent clinical trials (NCT02204943 and NCT02269982) [19, 37]. These alterations in osteoblastic gene regulators, including ALPL, RUNX2, SPP1, CDH11, and TNFSF11, were present in CTCs and not in paired cfDNA. Moreover, the genomic alterations in these genes were confirmed in both datasets in the same direction of genomic alteration (gain or loss) for each 
gene, suggesting that these genomic alterations may be important for metastasis to the bone. Furthermore, Cohen's kappa analysis of CTCs and cfDNA showed that AR has a discordance in the opposite direction, with greater detection in cfDNA than CTC DNA. This may be due to an increase in AR-expressing cells dying during hormonal therapy (abiraterone/enzalutamide). While the other CTC discordant genes may be the resistant, living clones that survive differentially and do not contribute as much to the cfDNA pool $[32,37]$, the $A R$-dependent genes may be more concordant (truncal) or discordant favoring cfDNA. Functional validation and mechanistic studies are needed to validate further and determine the clinical relevance of these CTC discordant genes to bone metastasis formation.

Our findings are limited by the small and exploratory nature of the present dataset and are considered hypothesis-generating. There are several key limitations of the present analysis. First, our sample size is small, and we lack sufficient power to conduct multivariable prognostic analyses of individual CTC-specific genomic alterations, adjusting for CTC enumeration and clinical prognostic factors, similar to what we have reported for AR-V7 [19]. Future studies will need to evaluate the independent prognostic utility of these CTC discordant alterations and characterize their functional relevance. Second, our CNA studies suggest that low tumor content will reduce the ability to detect such genomic alterations, either through low CTC enumeration or through low cfDNA tumor content, similar to findings by others $[6,8]$. However, our findings of CTC discordant alterations persisted even in samples with high tumor content, suggesting potentially real biologic divergence. Finally, DNA copy number alterations may not be associated with differences in mRNA or protein expression could be due to epigenetic repression, segment amplification, or partial gene amplification of chromatin regions that may influence mRNA expression, and single-copy differences may have little functional consequences vs. high-level gains or biallelic losses. To address this limitation, we examined CNA and mRNA expression in paired samples in public datasets and confirmed significant positive correlations between CNAs and mRNA for many key concordant and discordant 
genes observed in CTC DNA, such as gains in AR, MYC, FOXA1, KAT6B, and $K D M 6 A$, and losses in PTEN, RB1, and ZFHX3. Other discordant genes, such as MYCN, are already established to be critically relevant to small cell prostate cancer divergence [46]. Further mechanistic studies of CTC divergent genes are needed to evaluate their impact on treatment resistance and metastasis biology.

Our data establish that CTCs and cfDNA are valuable sources of genetic information that provide important, but potentially distinct and complementary information regarding tumor progression, treatment response/resistance, and metastasis biology. Additional larger studies of paired samples prospectively collected in clinically annotated datasets of patients with cancer in different disease states and during different treatments are needed to define whether CTCS, cfDNA, or a combined approach has greater clinical utility $[48,49]$. Our collective data suggest that CTCs harbor critical genomic information that may be missed by cfDNA analysis alone. Yet, reliance on CTC genomic data alone has limitations because many patients with metastatic prostate cancer lack detectable CTCs, particularly in earlier disease settings, limiting the clinical utility of this approach $[50,51]$. Therefore, analysis of both CTCs and cfDNA, as well as CTC or cell-free RNA and/or protein, could represent a valuable integrated approach. Our data support the need for the continued development of sensitive CTC DNA or RNA and cfDNA detection methods to identify clonal heterogeneity and the emergence of rare cells during the selection pressure of systemic therapies.

In summary, we find that while CTC DNA and cfDNA CNAs are largely concordant, a range of discordant findings in CTCs suggests that CTCs may report on divergent tumor evolution and heterogeneity that may be missed by cfDNA analysis. Genomic alterations in AR signaling, critical lineage oncogenes or tumor suppressors, epigenetic regulators, and osteomimicry pathways support a variety of progression and metastatic pathways important to CRPC lineage plasticity and bone metastasis 
biology. Efforts to functionally characterize these CTC-divergent genomic alterations and assess their clinical impact are critical.

\section{Acknowledgments}

We wish to acknowledge support from the Prostate Cancer Foundation and Bayer Pharmaceuticals. We wish to thank the Prostate Cancer Foundation and Movember for their financial support of this Global Treatment Sciences Challenge Award, and the US Department of Defense Prostate Cancer Clinical Trial Consortium for infrastructural support for this multicenter study. Dr. Armstrong was supported by a Prostate Cancer Foundation grant and the DCI P30 CA014236 as well as Duke Cancer Institute shared resources for biostatistics, Flow Cytometry, and Sequencing and Genomic Technologies. This work was partially funded by Department of Defense grants W81XWH-13-PCRP-CCA, W81XWH-17-2-0021 and W81XWH-14-20179 (DJG/AJA, Duke). JAS acknowledges support from the Department of Defense (PC170316). We acknowledge Dr. Emmanuel Antonarakis MD and Jun Luo Ph.D. for their contribution of CTC AR-V7 testing at Johns Hopkins, under W81XWH16-PCRP-CCRSA (E.S.A, Johns Hopkins). We acknowledge Drs. David Nanus and Scott Tagawa for their enrollment of patients at Weill Cornell Medical College under W81XWH-14-2-0159 (DMN, Weill Cornell). We wish to thank the study coordinators at Weill Cornell, Duke University, Johns Hopkins, University of Chicago, and Memorial Sloan Kettering Cancer Center and the DOD Prostate Cancer Clinical Trials Consortium (DOD PCCTC). Scott A. Tomlins reports that the University of Michigan has been issued patents on ETS gene fusions in prostate cancer, on which SAT is a coinventor. The diagnostic field of use was licensed to Hologic/Gen-Probe Inc., which has sublicensed rights to Roche/Ventana Medical Systems. SAT has served as a consultant for and received honoraria from Janssen, AbbVie, Sanofi, Almac Diagnostics, and Astellas/Medivation. SAT has had sponsored research agreements with Astellas/Medivation and GenomeDX. SAT is an equity holder in, prior consultant for, and current employee of Strata Oncology. We wish to 
acknowledge the dedication of our patients to provide blood samples at no clear benefit to them but for the benefits of all patients with prostate cancer. We acknowledge support from the Duke Cancer Institute Center for Prostate and Urologic Cancers. We would like to acknowledge the assistance of the Duke Molecular Physiology Institute Molecular Genomics core for the generation of data for the manuscript.

\section{References}

1. Ashworth T. A case of cancer in which cells similar to those in the tumours were seen in the blood after death. The Medical Journal of Australia. 1869;14:146-9. 2. Cristofanilli M, Budd GT, Ellis MJ, Stopeck A, Matera J, Miller MC, et al. Circulating tumor cells, disease progression, and survival in metastatic breast cancer. N Engl J Med. 2004;351(8):781-91. doi: 10.1056/NEJMoa040766. PubMed PMID: 15317891.

3. Parkinson DR, Dracopoli N, Petty BG, Compton C, Cristofanilli M, Deisseroth $A$, et al. Considerations in the development of circulating tumor cell technology for clinical use. J Transl Med. 2012;10:138. doi: 10.1186/1479-5876-10-138. PubMed PMID: 22747748; PubMed Central PMCID: PMCPMC3478228.

4. Siravegna G, Marsoni S, Siena S, Bardelli A. Integrating liquid biopsies into the management of cancer. Nat Rev Clin Oncol. 2017;14(9):531-48. doi: 10.1038/nrclinonc.2017.14. PubMed PMID: 28252003.

5. Zhang T, Armstrong AJ. Clinical Utility of Circulating Tumor Cells in Advanced Prostate Cancer. Curr Oncol Rep. 2016;18(1):3. doi: 10.1007/s11912-015-0490-9. PubMed PMID: 26700506.

6. de Bono JS, Scher HI, Montgomery RB, Parker C, Miller MC, Tissing $\mathrm{H}$, et al. Circulating tumor cells predict survival benefit from treatment in metastatic castration-resistant prostate cancer. Clin Cancer Res. 2008;14(19):6302-9. doi: 10.1158/1078-0432.CCR-08-0872. PubMed PMID: 18829513.

7. Schwarzenbach H, Hoon DS, Pantel K. Cell-free nucleic acids as biomarkers in cancer patients. Nat Rev Cancer. 2011;11(6):426-37. doi: 10.1038/nrc3066. PubMed PMID: 21562580.

8. Wyatt AW, Azad AA, Volik SV, Annala M, Beja K, McConeghy B, et al. Genomic Alterations in Cell-Free DNA and Enzalutamide Resistance in CastrationResistant Prostate Cancer. JAMA Oncol. 2016;2(12):1598-606. doi: 10.1001/jamaoncol.2016.0494. PubMed PMID: 27148695; PubMed Central PMCID: PMCPMC5097690.

9. Torga G, Pienta KJ. Patient-Paired Sample Congruence Between 2 Commercial Liquid Biopsy Tests. JAMA Oncol. 2018;4(6):868-70. doi: 
10.1001/jamaoncol.2017.4027. PubMed PMID: 29242909; PubMed Central PMCID: PMCPMC6145681.

10. Goodall J, Mateo J, Yuan W, Mossop H, Porta N, Miranda S, et al. Circulating Cell-Free DNA to Guide Prostate Cancer Treatment with PARP Inhibition. Cancer Discov. 2017;7(9):1006-17. doi: 10.1158/2159-8290.CD-17-0261. PubMed PMID: 28450425.

11. Lambros MB, Seed G, Sumanasuriya S, Gil V, Crespo M, Fontes M, et al. Single-Cell Analyses of Prostate Cancer Liquid Biopsies Acquired by Apheresis. Clin Cancer Res. 2018. doi: 10.1158/1078-0432.CCR-18-0862. PubMed PMID: 30093450.

12. Wyatt AW, Annala M, Aggarwal R, Beja K, Feng F, Youngren J, et al. Concordance of Circulating Tumor DNA and Matched Metastatic Tissue Biopsy in Prostate Cancer. J Natl Cancer Inst. 2017;109(12). doi: 10.1093/jnci/djx118. PubMed PMID: 29206995.

13. Quigley D, Alumkal JJ, Wyatt AW, Kothari V, Foye A, Lloyd P, et al. Analysis of Circulating Cell-Free DNA Identifies Multiclonal Heterogeneity of BRCA2 Reversion Mutations Associated with Resistance to PARP Inhibitors. Cancer Discov. 2017;7(9):999-1005. doi: 10.1158/2159-8290.CD-17-0146. PubMed PMID: 28450426; PubMed Central PMCID: PMCPMC5581695.

14. Scher HI, Fizazi K, Saad F, Taplin ME, Sternberg CN, Miller K, et al. Increased survival with enzalutamide in prostate cancer after chemotherapy. N Engl J Med. 2012;367(13):1187-97. doi: 10.1056/NEJMoa1207506. PubMed PMID: 22894553.

15. de Bono JS, Chowdhury S, Feyerabend S, Elliott T, Grande E, MelhemBertrandt A, et al. Antitumour Activity and Safety of Enzalutamide in Patients with Metastatic Castration-resistant Prostate Cancer Previously Treated with Abiraterone Acetate Plus Prednisone for $>/=24$ weeks in Europe. Eur Urol. 2018;74(1):37-45. doi: 10.1016/j.eururo.2017.07.035. PubMed PMID: 28844372.

16. Antonarakis ES, Lu C, Wang H, Luber B, Nakazawa M, Roeser JC, et al. AR$\mathrm{V} 7$ and resistance to enzalutamide and abiraterone in prostate cancer. $\mathrm{N}$ Engl $\mathrm{J}$ Med. 2014;371(11):1028-38. doi: 10.1056/NEJMoa1315815. PubMed PMID: 25184630; PubMed Central PMCID: PMCPMC4201502.

17. Scher HI, Lu D, Schreiber NA, Louw J, Graf RP, Vargas HA, et al. Association of AR-V7 on Circulating Tumor Cells as a Treatment-Specific Biomarker With Outcomes and Survival in Castration-Resistant Prostate Cancer. JAMA Oncol. 2016. doi: 10.1001/jamaoncol.2016.1828. PubMed PMID: 27262168.

18. Scher HI, Graf RP, Schreiber NA, Jayaram A, Winquist E, McLaughlin B, et al. Assessment of the Validity of Nuclear-Localized Androgen Receptor Splice Variant 7 in Circulating Tumor Cells as a Predictive Biomarker for Castration-Resistant Prostate Cancer. JAMA Oncol. 2018;4(9):1179-86. doi: 10.1001/jamaoncol.2018.1621. PubMed PMID: 29955787; PubMed Central PMCID: PMCPMC6139066.

19. Armstrong AJ, Halabi S, Luo J, Nanus DM, Giannakakou P, Szmulewitz RZ, et al. Prospective Multicenter Validation of Androgen Receptor Splice Variant 7 and 
Hormone Therapy Resistance in High-Risk Castration-Resistant Prostate Cancer: The PROPHECY Study. J Clin Oncol. 2019:JCO1801731. doi: 10.1200/JCO.18.01731. PubMed PMID: 30865549.

20. De Laere B, Oeyen S, Mayrhofer M, Whitington T, van Dam PJ, Van Oyen P, et al. TP53 Outperforms Other Androgen Receptor Biomarkers to Predict Abiraterone or Enzalutamide Outcome in Metastatic Castration-Resistant Prostate Cancer. Clin Cancer Res. 2019;25(6):1766-73. doi: 10.1158/1078-0432.CCR-181943. PubMed PMID: 30209161; PubMed Central PMCID: PMCPMC6330086.

21. Armstrong AJ, Eisenberger MA, Halabi S, Oudard S, Nanus DM, Petrylak DP, et al. Biomarkers in the management and treatment of men with metastatic castration-resistant prostate cancer. Eur Urol. 2012;61(3):549-59. doi: 10.1016/j.eururo.2011.11.009. PubMed PMID: 22099611; PubMed Central PMCID: PMCPMC3445625.

22. Taylor BS, Schultz N, Hieronymus H, Gopalan A, Xiao Y, Carver BS, et al. Integrative genomic profiling of human prostate cancer. Cancer Cell. 2010;18(1):1122. doi: 10.1016/j.ccr.2010.05.026. PubMed PMID: 20579941; PubMed Central PMCID: PMCPMC3198787.

23. Terada N, Akamatsu S, Kobayashi T, Inoue T, Ogawa O, Antonarakis ES. Prognostic and predictive biomarkers in prostate cancer: latest evidence and clinical implications. Ther Adv Med Oncol. 2017;9(8):565-73. doi:

10.1177/1758834017719215. PubMed PMID: 28794807; PubMed Central PMCID: PMCPMC5524249.

24. Hodara E, Morrison G, Cunha A, Zainfeld D, Xu T, Xu Y, et al. Multiparametric liquid biopsy analysis in metastatic prostate cancer. JCI Insight. 2019;4(5). doi: 10.1172/jci.insight.125529. PubMed PMID: 30702443.

25. Hong CS, Singh LN, Mullikin JC, Biesecker LG. Assessing the reproducibility of exome copy number variations predictions. Genome Med. 2016;8(1):82. doi: 10.1186/s13073-016-0336-6. PubMed PMID: 27503473; PubMed Central PMCID: PMCPMC4976506.

26. Provencio M, Perez-Callejo D, Torrente M, Martin P, Calvo V, Gutierrez L, et al. Concordance between circulating tumor cells and clinical status during follow-up in anaplastic lymphoma kinase (ALK) non-small-cell lung cancer patients.

Oncotarget. 2017;8(35):59408-16. doi: 10.18632/oncotarget.19722. PubMed PMID: 28938646; PubMed Central PMCID: PMCPMC5601742.

27. Romanel A, Gasi Tandefelt D, Conteduca V, Jayaram A, Casiraghi N, Wetterskog D, et al. Plasma AR and abiraterone-resistant prostate cancer. Sci Transl Med. 2015;7(312):312re10. doi: 10.1126/scitransImed.aac9511. PubMed PMID: 26537258.

28. Ulz P, Belic J, Graf R, Auer M, Lafer I, Fischereder K, et al. Whole-genome plasma sequencing reveals focal amplifications as a driving force in metastatic prostate cancer. Nat Commun. 2016;7:12008. doi: 10.1038/ncomms12008. PubMed PMID: 27328849; PubMed Central PMCID: PMCPMC4917969.

29. Massard C, Oulhen M, Le Moulec S, Auger N, Foulon S, Abou-Lovergne A, et al. Phenotypic and genetic heterogeneity of tumor tissue and circulating tumor cells 
in patients with metastatic castration-resistant prostate cancer: A report from the PETRUS prospective study. Oncotarget. 2016;7(34):55069-82. doi:

10.18632/oncotarget.10396. PubMed PMID: 27391263; PubMed Central PMCID: PMCPMC5342402.

30. Andrew John Armstrong M, ScM, Halabi S, Luo J, David Nanus M, Paraskevi Giannakakou P, Russell Szmulewitz M, et al. Prospective multicenter validation of AR-V7 and hormone therapy resistance in high risk castration resistant prostate cancer: the PROPHECY study. Journal of Clinical Oncology. 2019.

31. Scher HI, Halabi S, Tannock I, Morris M, Sternberg CN, Carducci MA, et al. Design and end points of clinical trials for patients with progressive prostate cancer and castrate levels of testosterone: recommendations of the Prostate Cancer Clinical Trials Working Group. J Clin Oncol. 2008;26(7):1148-59. doi:

10.1200/JCO.2007.12.4487. PubMed PMID: 18309951; PubMed Central PMCID: PMCPMC4010133.

32. Gupta S, Li J, Kemeny G, Bitting RL, Beaver J, Somarelli JA, et al. Whole Genomic Copy Number Alterations in Circulating Tumor Cells from Men with Abiraterone or Enzalutamide-Resistant Metastatic Castration-Resistant Prostate Cancer. Clin Cancer Res. 2017;23(5):1346-57. doi: 10.1158/1078-0432.CCR-161211. PubMed PMID: 27601596.

33. Scher HI, Lu D, Schreiber NA, Louw J, Graf RP, Vargas HA, et al. Association of AR-V7 on Circulating Tumor Cells as a Treatment-Specific Biomarker With Outcomes and Survival in Castration-Resistant Prostate Cancer. JAMA Oncol. 2016;2(11):1441-9. Epub 2016/06/05. doi: 10.1001/jamaoncol.2016.1828. PubMed PMID: 27262168; PubMed Central PMCID: PMCPmc5206761.

34. Scher HI, Graf RP, Schreiber NA, McLaughlin B, Lu D, Louw J, et al. Nuclearspecific AR-V7 Protein Localization is Necessary to Guide Treatment Selection in Metastatic Castration-resistant Prostate Cancer. Eur Urol. 2017;71(6):874-82. doi: 10.1016/j.eururo.2016.11.024. PubMed PMID: 27979426; PubMed Central PMCID: PMCPMC5401782.

35. Gao J, Aksoy BA, Dogrusoz U, Dresdner G, Gross B, Sumer SO, et al. Integrative analysis of complex cancer genomics and clinical profiles using the cBioPortal. Sci Signal. 2013;6(269):pl1. doi: 10.1126/scisignal.2004088. PubMed PMID: 23550210; PubMed Central PMCID: PMCPMC4160307.

36. Hovelson DH, Liu CJ, Wang Y, Kang Q, Henderson J, Gursky A, et al. Rapid, ultra low coverage copy number profiling of cell-free DNA as a precision oncology screening strategy. Oncotarget. 2017;8(52):89848-66. doi:

10.18632/oncotarget.21163. PubMed PMID: 29163793; PubMed Central PMCID: PMCPMC5685714.

37. Armstrong AJ, Gupta S, Healy P, Kemeny G, Leith B, Zalutsky MR, et al. Pharmacodynamic study of radium-223 in men with bone metastatic castration resistant prostate cancer. PLoS One. 2019;14(5):e0216934. doi:

10.1371/journal.pone.0216934. PubMed PMID: 31136607; PubMed Central PMCID: PMCPMC6538141 University from Bayer and serve as speakers for Bayer.

Competing interest statement: Drs. George and Armstrong receive consulting and 
speaking income from Bayer as well as research funding (to Duke University) from Bayer. No other conflicts of interest to report. The funders had no role in study design, data collection and analysis, decision to publish, or preparation of the manuscript.this does not alter our adherence to PLOS ONE policies on sharing data and materials.

38. Robinson D, Van Allen EM, Wu YM, Schultz N, Lonigro RJ, Mosquera JM, et al. Integrative clinical genomics of advanced prostate cancer. Cell. 2015;161(5):1215-28. doi: 10.1016/j.cell.2015.05.001. PubMed PMID: 26000489; PubMed Central PMCID: PMCPMC4484602.

39. Scher HI, Graf RP, Schreiber NA, McLaughlin B, Jendrisak A, Wang Y, et al. Phenotypic Heterogeneity of Circulating Tumor Cells Informs Clinical Decisions between AR Signaling Inhibitors and Taxanes in Metastatic Prostate Cancer. Cancer Res. 2017;77(20):5687-98. doi: 10.1158/0008-5472.CAN-17-1353. PubMed PMID: 28819021; PubMed Central PMCID: PMCPMC5666339.

40. Beltran H, Jendrisak A, Landers M, Mosquera JM, Kossai M, Louw J, et al. The Initial Detection and Partial Characterization of Circulating Tumor Cells in Neuroendocrine Prostate Cancer. Clin Cancer Res. 2016;22(6):1510-9. doi: 10.1158/1078-0432.CCR-15-0137. PubMed PMID: 26671992; PubMed Central PMCID: PMCPMC4990782.

41. Koeneman KS, Yeung F, Chung LW. Osteomimetic properties of prostate cancer cells: a hypothesis supporting the predilection of prostate cancer metastasis and growth in the bone environment. Prostate. 1999;39(4):246-61. PubMed PMID: 10344214.

42. Kumar A, Coleman I, Morrissey C, Zhang X, True LD, Gulati R, et al. Substantial interindividual and limited intraindividual genomic diversity among tumors from men with metastatic prostate cancer. Nat Med. 2016;22(4):369-78. doi: 10.1038/nm.4053. PubMed PMID: 26928463; PubMed Central PMCID: PMCPMC5045679.

43. Hong MK, Macintyre G, Wedge DC, Van Loo P, Patel K, Lunke S, et al. Tracking the origins and drivers of subclonal metastatic expansion in prostate cancer. Nat Commun. 2015;6:6605. doi: 10.1038/ncomms7605. PubMed PMID: 25827447; PubMed Central PMCID: PMCPMC4396364.

44. Torquato S, Pallavajjala A, Goldstein A, Toro PV, Silberstein JL, Lee J, et al. Genetic Alterations Detected in Cell-Free DNA Are Associated With Enzalutamide and Abiraterone Resistance in Castration-Resistant Prostate Cancer. JCO Precision Oncology. 2019;(3):1-14. doi: 10.1200/po.18.00227.

45. Mosquera JM, Beltran H, Park K, MacDonald TY, Robinson BD, Tagawa ST, et al. Concurrent AURKA and MYCN gene amplifications are harbingers of lethal treatment-related neuroendocrine prostate cancer. Neoplasia. 2013;15(1):1-10. PubMed PMID: 23358695; PubMed Central PMCID: PMCPMC3556934.

46. Beltran H, Rickman DS, Park K, Chae SS, Sboner A, MacDonald TY, et al. Molecular characterization of neuroendocrine prostate cancer and identification of new drug targets. Cancer Discov. 2011;1(6):487-95. doi: 10.1158/2159-8290.CD-110130. PubMed PMID: 22389870; PubMed Central PMCID: PMCPMC3290518. 
47. Beltran H, Prandi D, Mosquera JM, Benelli M, Puca L, Cyrta J, et al. Divergent clonal evolution of castration-resistant neuroendocrine prostate cancer. Nat Med. 2016;22(3):298-305. doi: 10.1038/nm.4045. PubMed PMID: 26855148; PubMed Central PMCID: PMCPMC4777652.

48. Ilie M, Hofman V, Long E, Bordone O, Selva E, Washetine K, et al. Current challenges for detection of circulating tumor cells and cell-free circulating nucleic acids, and their characterization in non-small cell lung carcinoma patients. What is the best blood substrate for personalized medicine? Ann Transl Med. 2014;2(11):107. doi: 10.3978/j.issn.2305-5839.2014.08.11. PubMed PMID: 25489581; PubMed Central PMCID: PMCPMC4245510.

49. Chemi F, Rothwell DG, McGranahan N, Gulati S, Abbosh C, Pearce SP, et al. Pulmonary venous circulating tumor cell dissemination before tumor resection and disease relapse. Nat Med. 2019. Epub 2019/10/09. doi: 10.1038/s41591-019-05931. PubMed PMID: 31591595.

50. de Bono JS, Attard G, Adjei A, Pollak MN, Fong PC, Haluska P, et al. Potential applications for circulating tumor cells expressing the insulin-like growth factor-I receptor. Clin Cancer Res. 2007;13(12):3611-6. doi: 10.1158/10780432.CCR-07-0268. PubMed PMID: 17575225.

51. Scher HI, Heller G, Molina A, Attard G, Danila DC, Jia X, et al. Circulating tumor cell biomarker panel as an individual-level surrogate for survival in metastatic castration-resistant prostate cancer. J Clin Oncol. 2015;33(12):1348-55. doi: 10.1200/JCO.2014.55.3487. PubMed PMID: 25800753; PubMed Central PMCID: PMCPMC4397279. 
20 men with mCRPC treated with radium-223 (NCT02204943)

(Prior or concurrent therapy with either abiraterone acetate or enzalutamide)

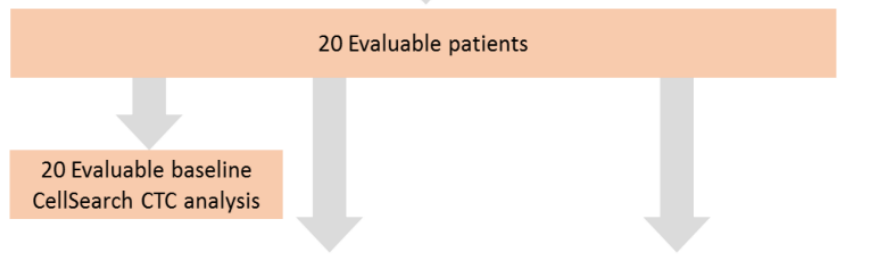

37 CTCs (EpCAM+, CD45-) from 19 men (14 baseline and 23 longitudinal) Copy number analysis (CGH)

40 cfDNA samples from 18 men (18 baseline and 22 longitudinal) Copy number analysis (CGH)

Genomic Concordance/Discordance in CNA from

32 paired CTC and cfDNA from 16 men (14 baseline and 18 longitudinal)

\section{0 men with $\mathrm{mCRPC}$}

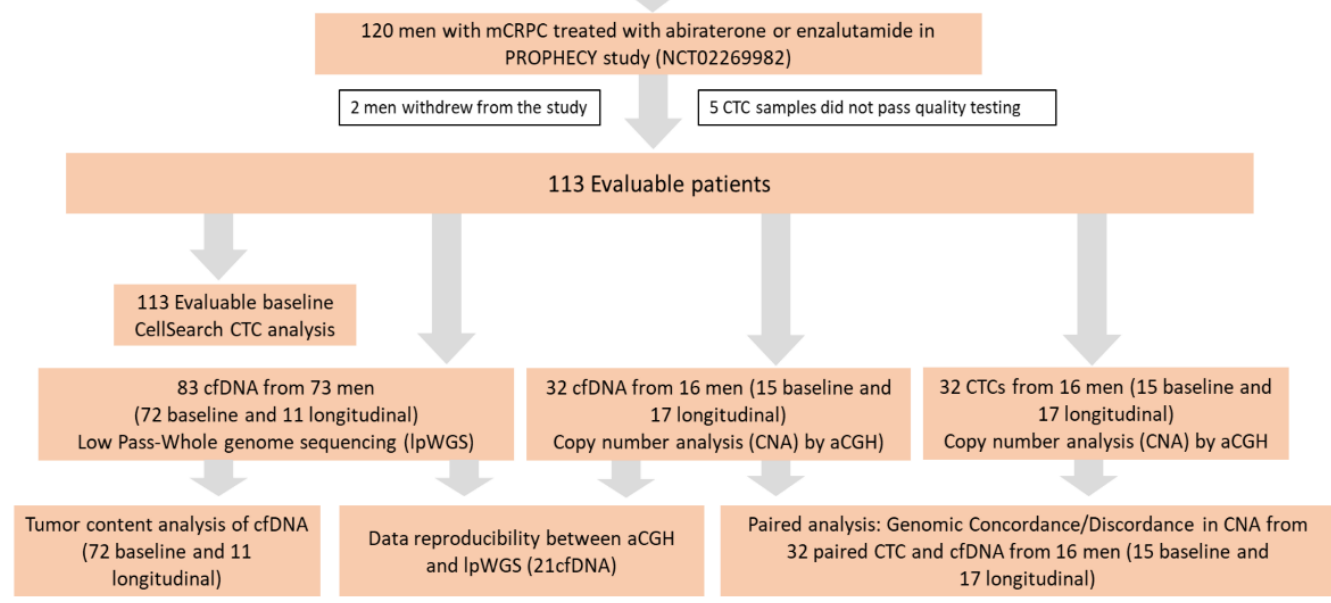

This article is protected by copyright. All rights reserved. 


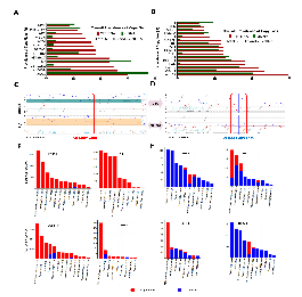

This article is protected by copyright. All rights reserved. 


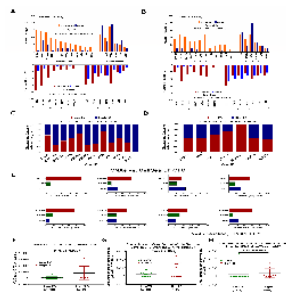

This article is protected by copyright. All rights reserved. 


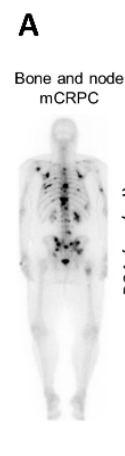

B

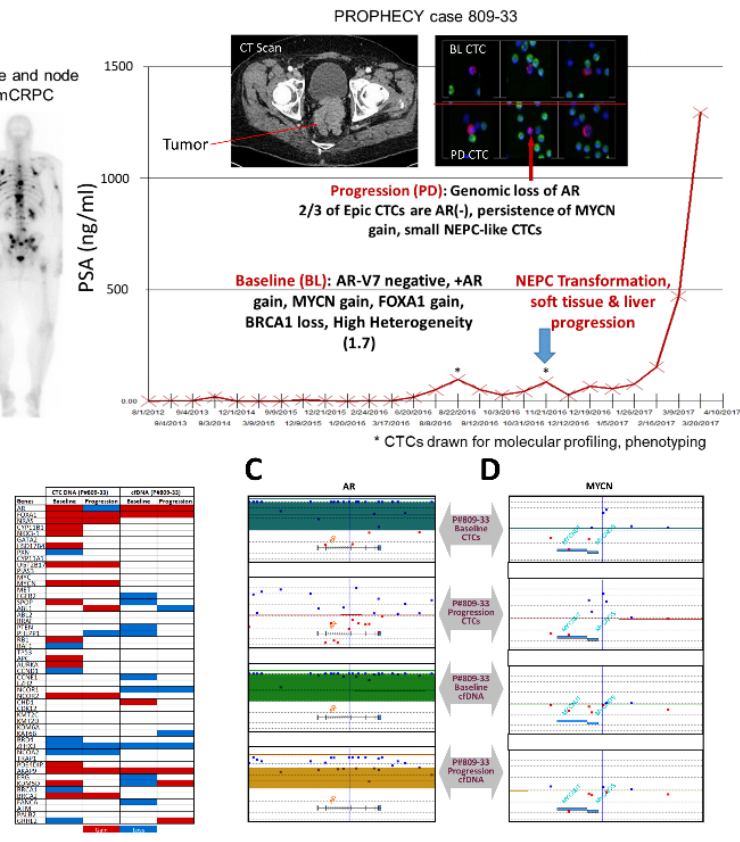

PROPHECY case 809-33
E

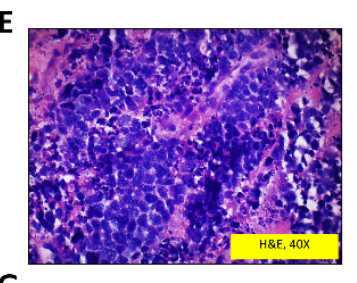

G

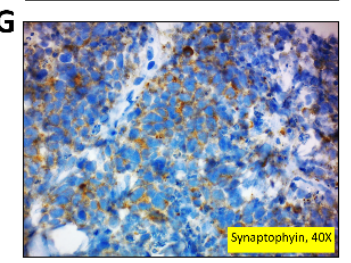

$H$
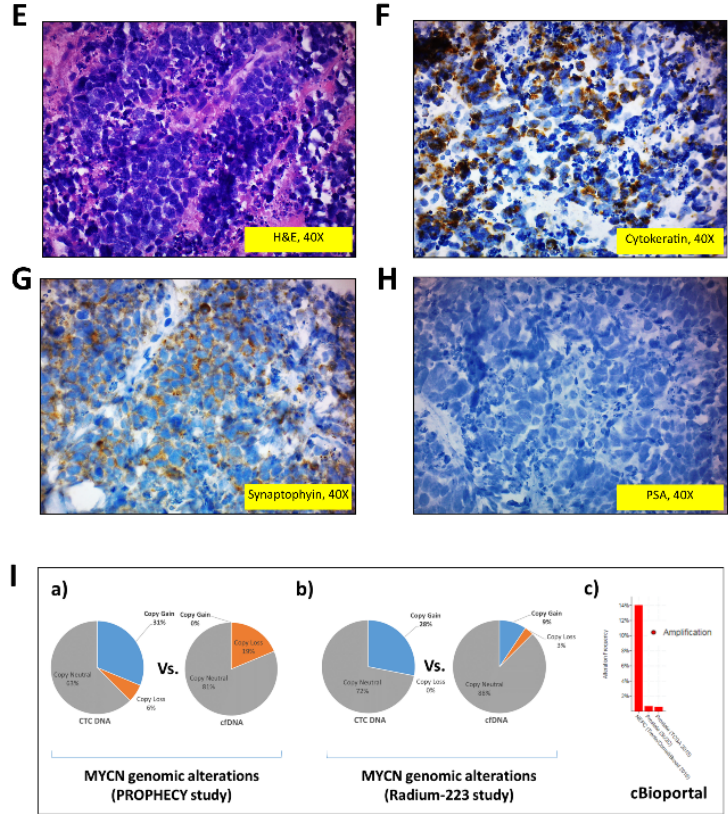

This article is protected by copyright. All rights reserved. 
A

Kaplan-Meier PFS Curves by JHU AR-V7

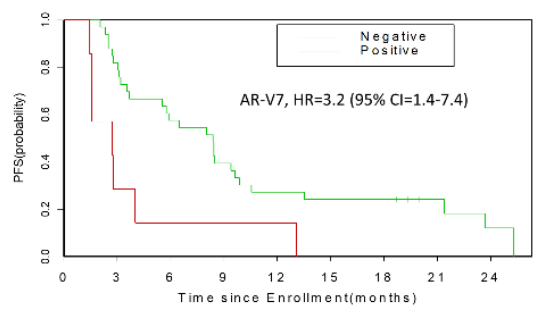

Patients at Risk
Negative
Pogitis

C

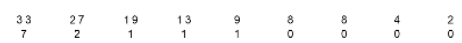

Kaplan-Meier PFS Curves by Pten Loss

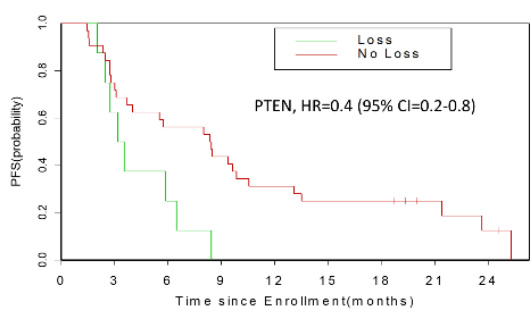

Pationts at Rish
Loss
No Loss

$\begin{array}{lllllllll}8 & 54 & 2 & 0 & 0 & 0 & 0 & 0 & 0 \\ 32 & 24 & 18 & 14 & 10 & 8 & 8 & 4 & 2\end{array}$

E

Kaplan-Meier PFS Curves by BRCA $2 \mathrm{G}$ ain

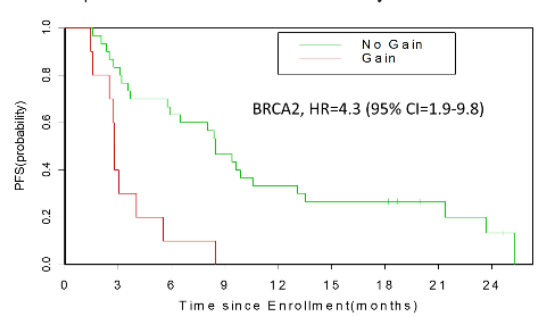

Patlents at Ris
Nog gin
Gain

$\begin{array}{lllllllll}30 & 25 & 19 & 14 & 10 & 8 & 8 & 4 & 2 \\ 10 & 4 & 1 & 0 & 0 & 0 & 0 & 0 & 0\end{array}$
B
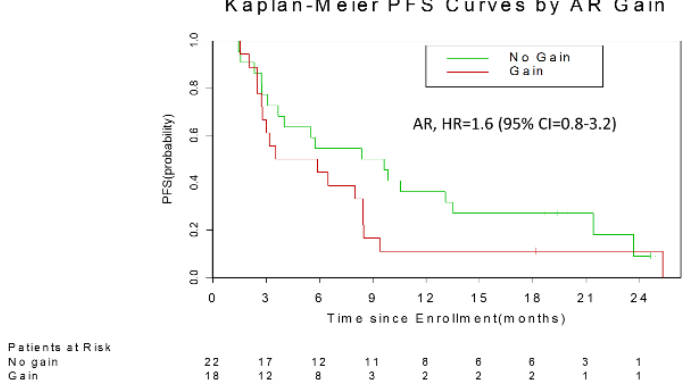

$\begin{array}{lllllllll}22 & 17 & 12 & 11 & 8 & 6 & 6 & 3 & 1 \\ 18 & 12 & 8 & 3 & 2 & 2 & 2 & 1 & 1\end{array}$

D

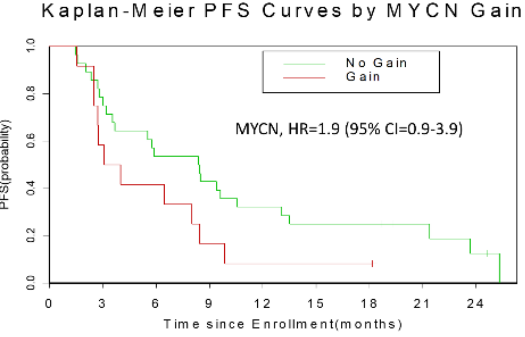

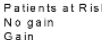

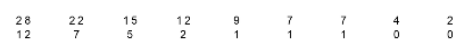

Kaplan-Meier PFS Curves by RUNX2 Loss

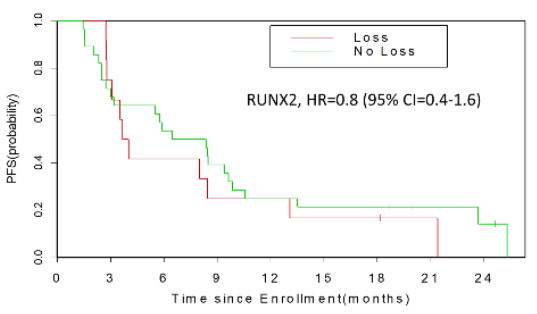

Patients at Risk
Loss $\begin{array}{lllllllll}12 & 9 & 5 & 3 & 3 & 2 & 2 & \frac{1}{28} & 0 \\ 28 & 20 & 15 & 11 & 7 & 6 & 6 & 3 & 2\end{array}$ 


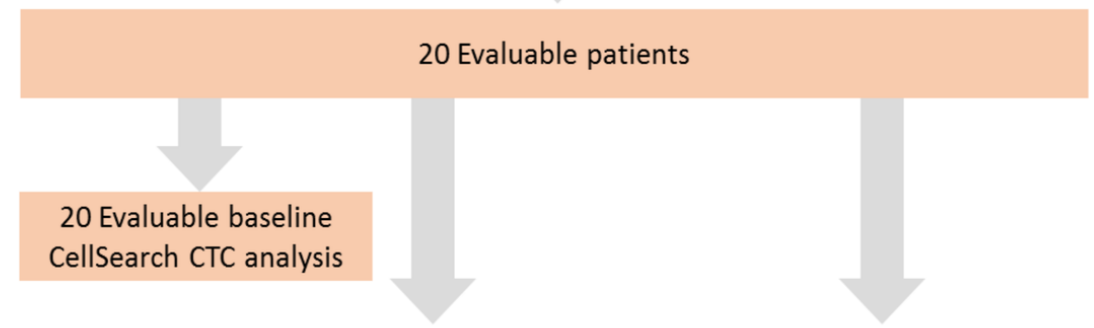

37 CTCs (EpCAM+, CD45-) from 19 men (14 baseline and 23 longitudinal) Copy number analysis (CGH)

40 cfDNA samples from 18 men

(18 baseline and 22 longitudinal)

Copy number analysis (CGH)

Genomic Concordance/Discordance in CNA from 32 paired CTC and cfDNA from 16 men (14 baseline and 18 longitudinal)

\section{0 men with mCRPC} PROPHECY study (NCT02269982)

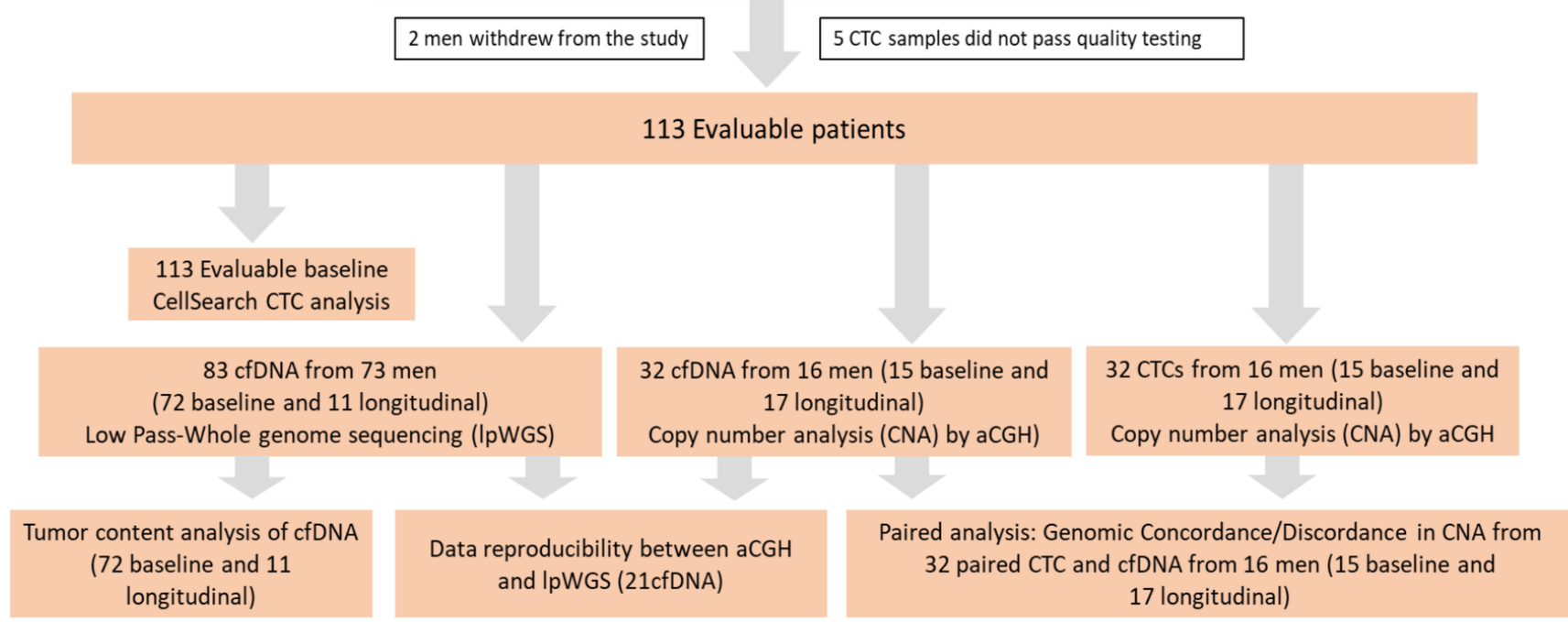

\section{GCC_22824_Figure 1.tif}



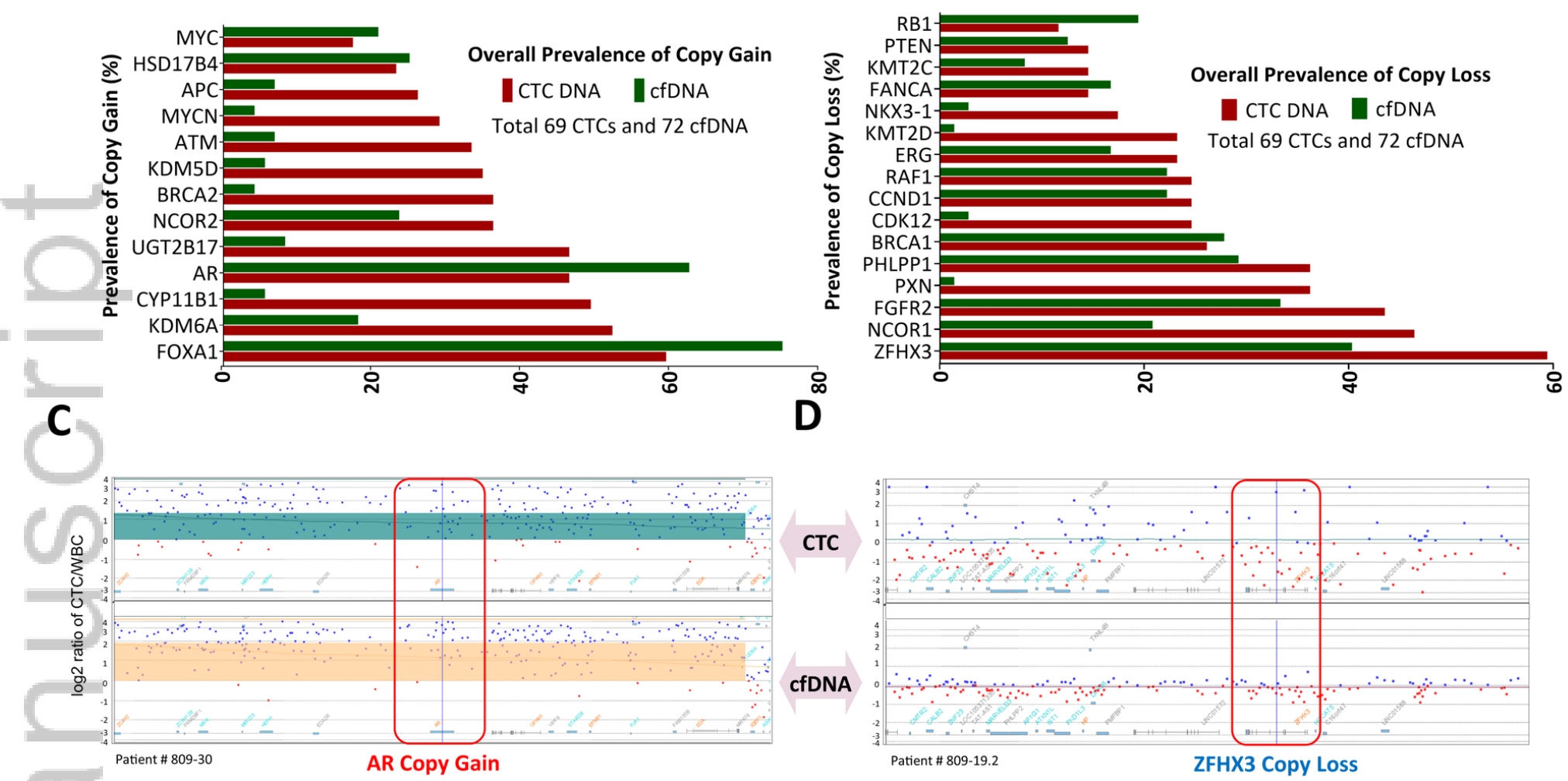

E
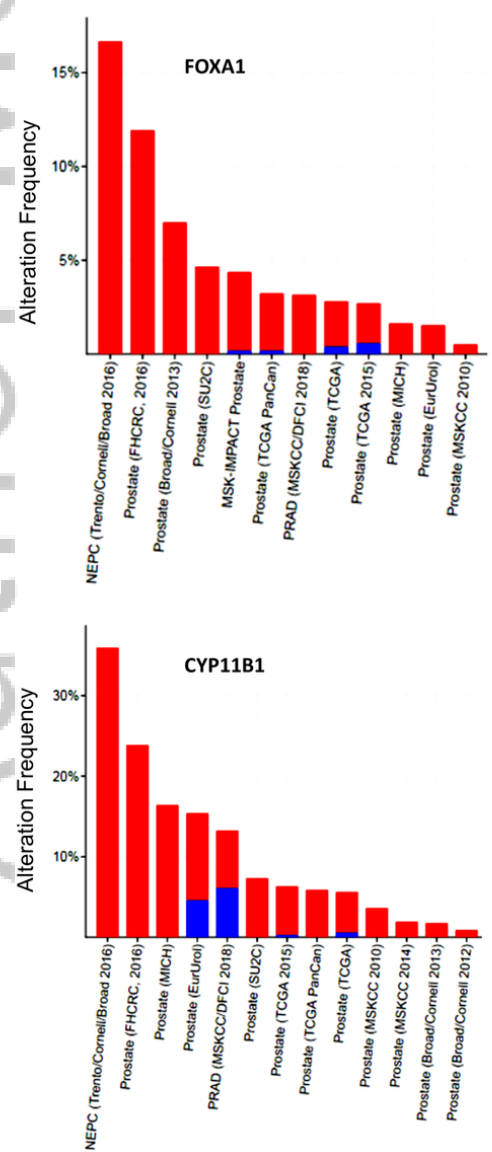
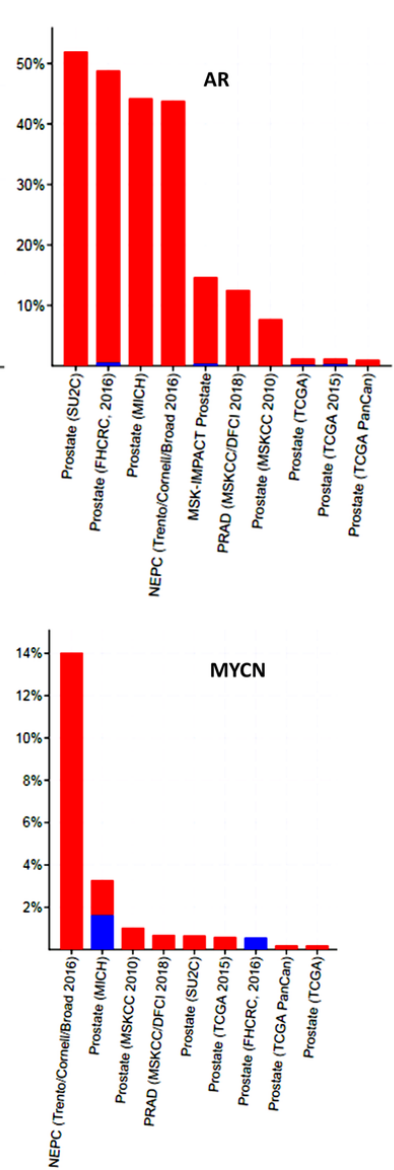

F
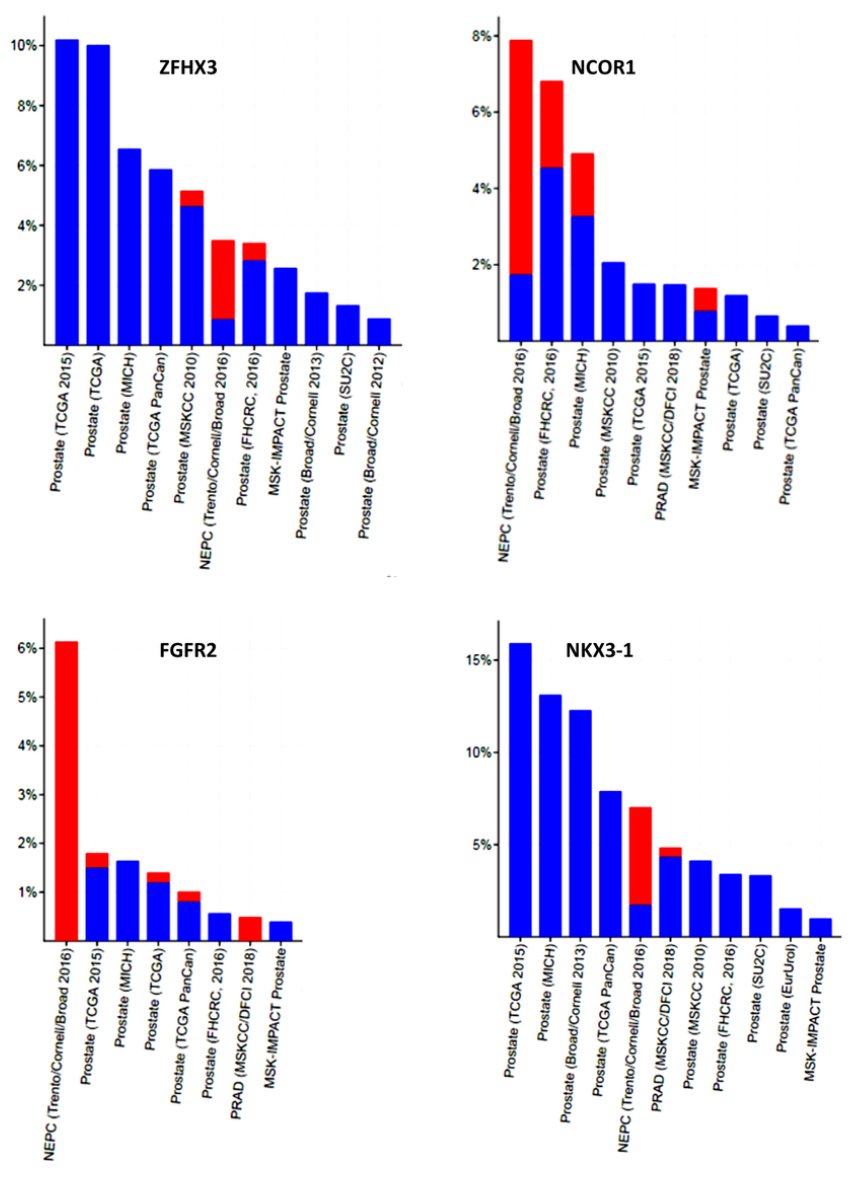

GCC_22824_Figure 2.tif

This article is protected by copyright. All rights reserved. 
A
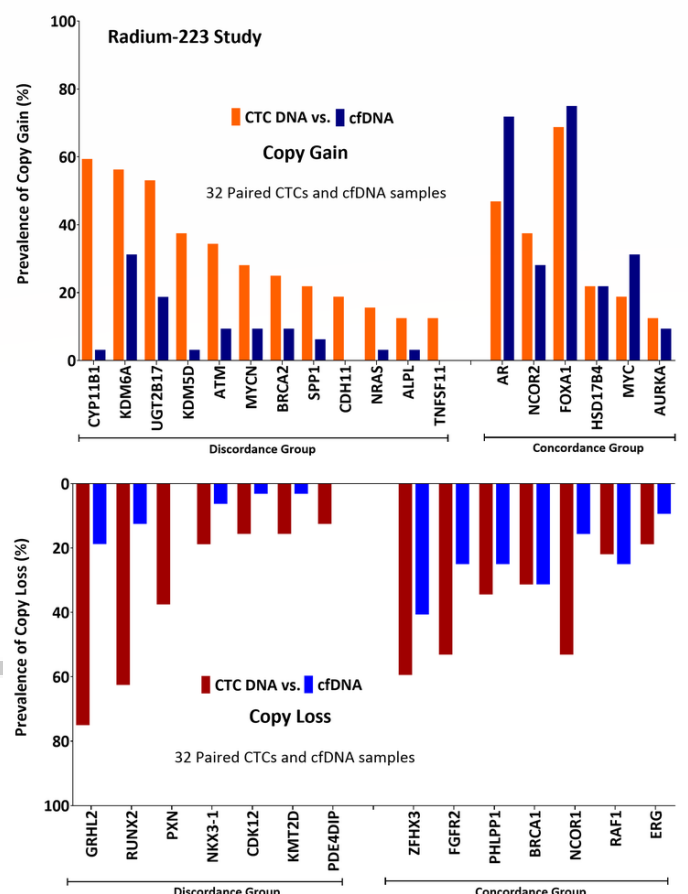

C

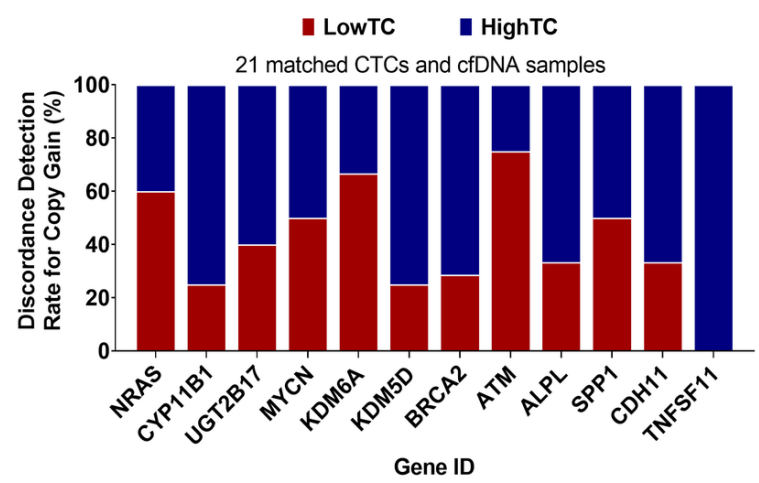

B
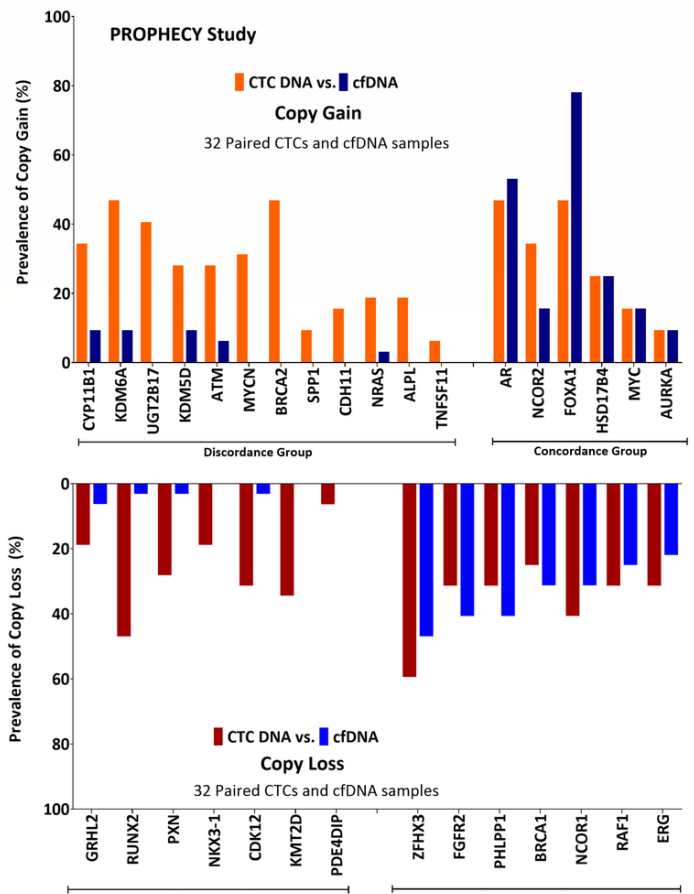

D

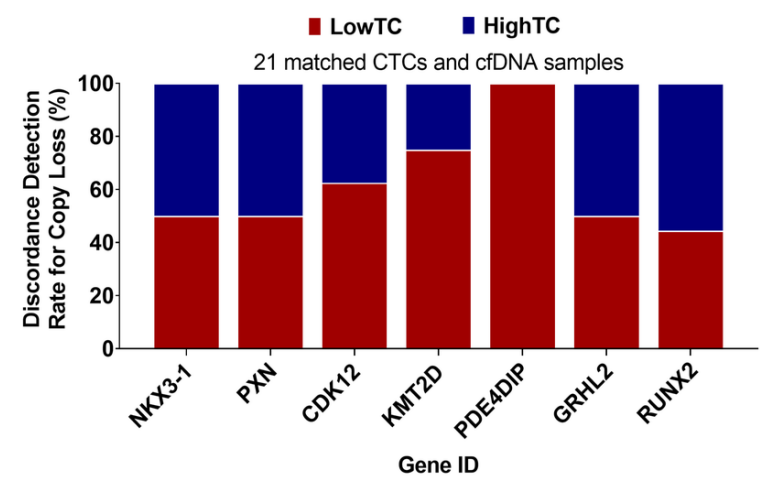

E

CNAs vs. CellSearch CTC
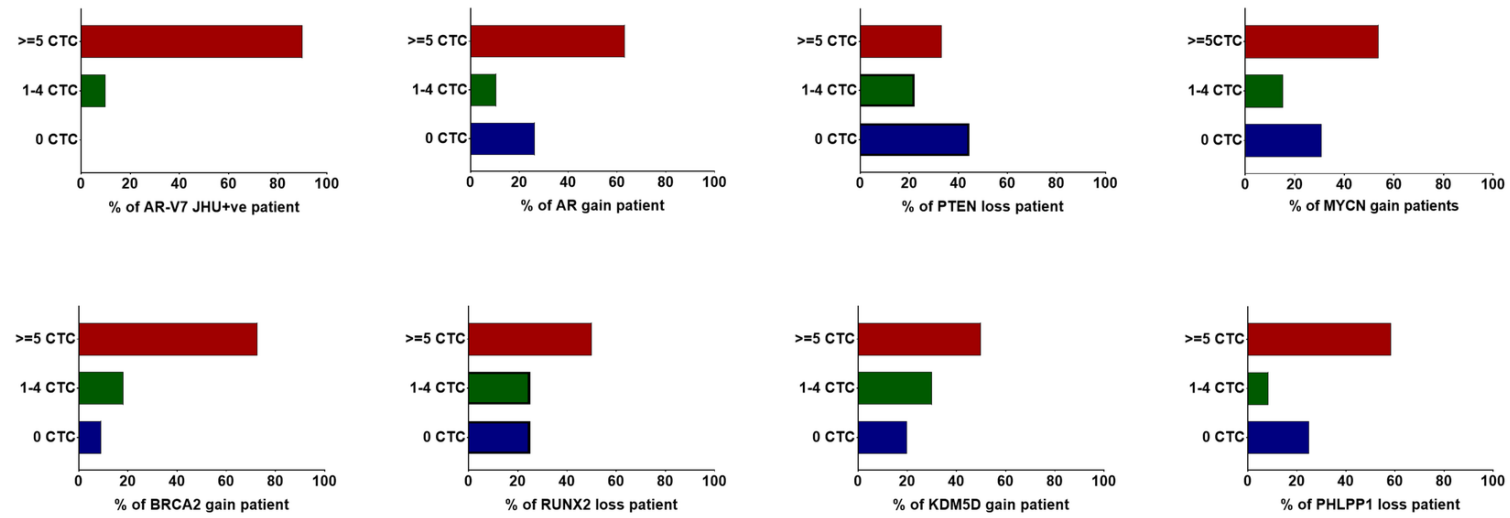

$\mathrm{N}=45$ baseline, $\mathrm{PROPHECY}$

G

Tumor Content vs. CTC (CellSearch)

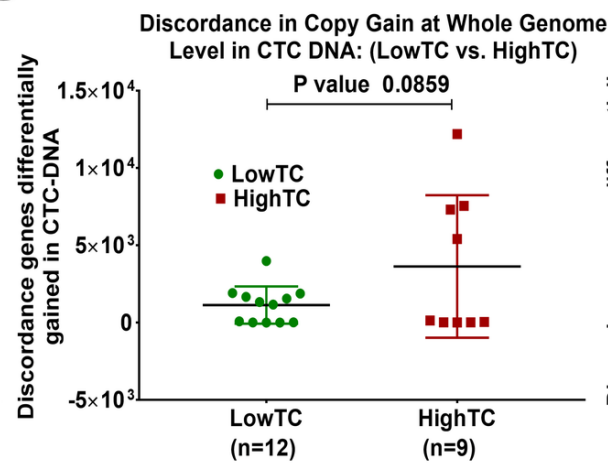

$\mathrm{H}$

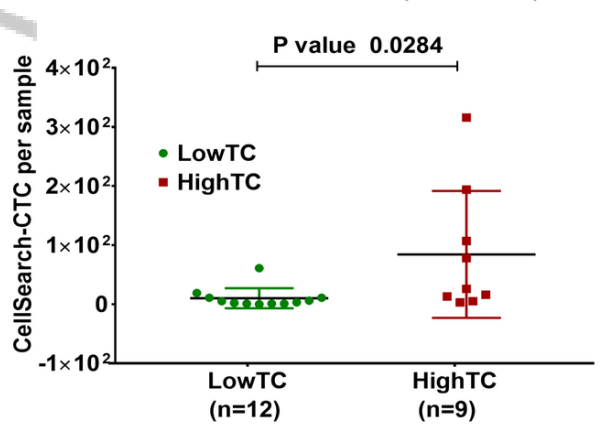

Discordance in Copy Gain at Whole Genome Level in cfDNA: (LowTC vs. HighTC)

GCC_22824_Figure 3.tif

This article is protected by copyright. All rights reserved. 
A

Kaplan-Meier PFS Curves by JHU AR-V7

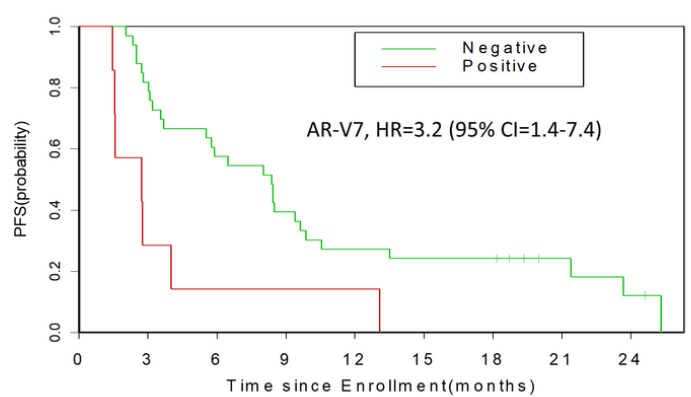

$\begin{array}{ccccccccc}33 & 27 & 19 & 13 & 9 & 8 & 8 & 4 & 2 \\ 7 & 2 & 1 & 1 & 1 & 0 & 0 & 0 & 0\end{array}$

Kaplan-Meier PFS Curves by Pten Loss

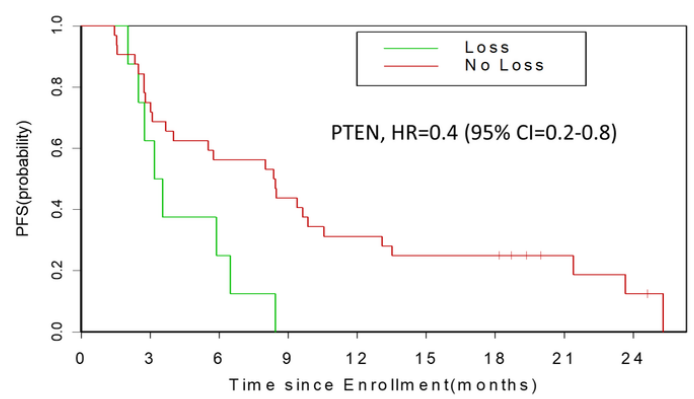

Patients at $R$ is Loss

\section{$E$}

Kaplan-Meier PFS Curves by BRCA2 Gain

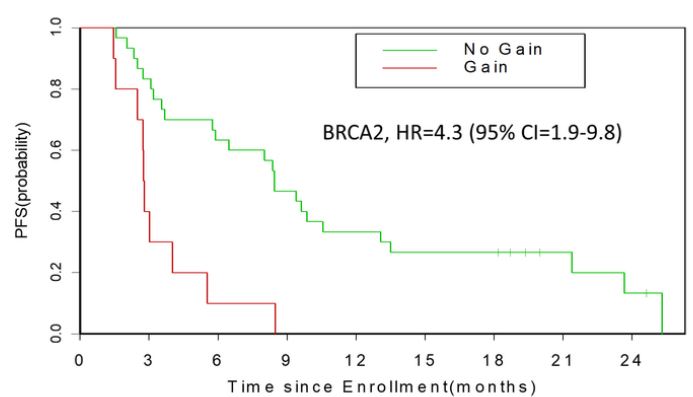

30
10
B

Kaplan-Meier PFS Curves by AR Gain

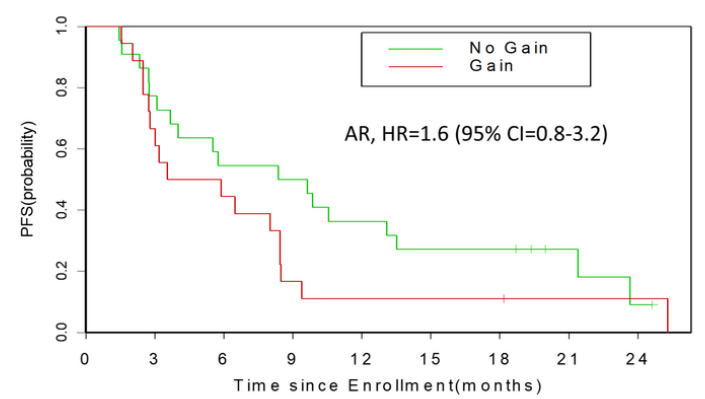

Patients at $R$ isk No ga in

D

Kaplan-Meier PFS Curves by MYCN Gain

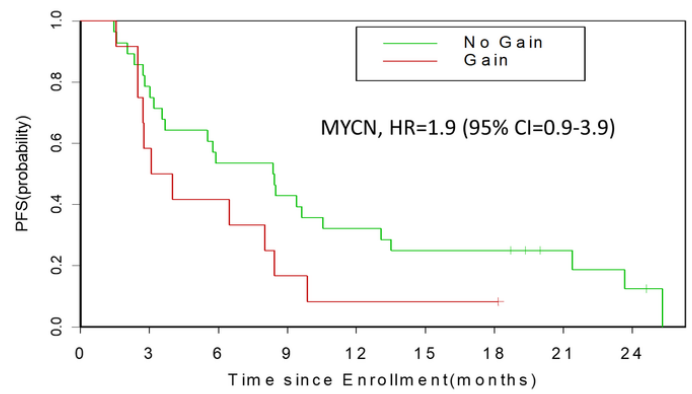

$P$ atients at $R$ is $k$ Nogain

$\mathbf{F}$ Kaplan-Meier PFS Curves by RUNX2 Loss

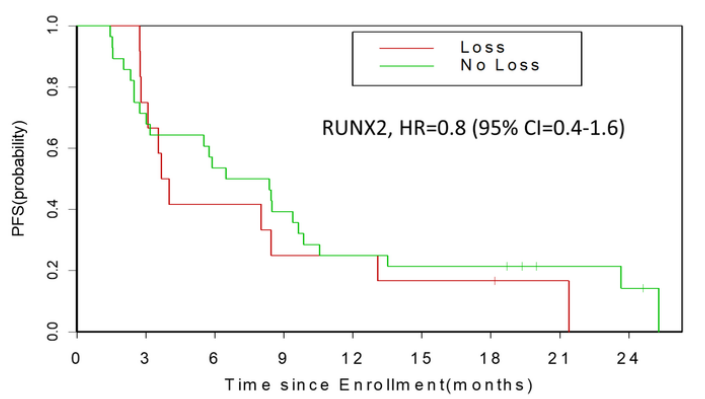

Patients at $R$ isk Loss
No Loss $\begin{array}{ccccccccc}28 & 22 & 15 & 12 & 9 & 7 & 7 & 4 & 2 \\ 12 & 7 & 5 & 2 & 1 & 1 & 1 & 0 & 0\end{array}$

$\begin{array}{lllllllll}12 & 9 & 5 & 3 & 3 & 2 & 2 & 1 & 0 \\ 28 & 20 & 15 & 11 & 7 & 6 & 6 & 3 & 2\end{array}$

GCC_22824_Figure 5.tif 